\title{
Development and evaluation of a new modular nanotransporter for drug delivery into nuclei of pathological cells expressing folate receptors
}

This article was published in the following Dove Press journal:

Drug Design, Development and Therapy

26 April 2017

Number of times this article has been viewed

\author{
Tatiana A Slastnikova' \\ Andrey A Rosenkranz ${ }^{1,2}$ \\ Yuri V Khramtsov' \\ Tatiana S Karyagina' \\ Sergey A Ovechko \\ Alexander S Sobolev ${ }^{1,2}$ \\ 'Laboratory of Molecular Genetics \\ of Intracellular Transport, Institute \\ of Gene Biology, Russian Academy of \\ Sciences, ${ }^{2}$ Department of Biophysics, \\ Faculty of Biology, Lomonosov \\ Moscow State University, \\ Moscow, Russia
}

Purpose: Modular nanotransporters (MNTs) are artificial multifunctional systems designed to facilitate receptor-specific transport from the cell surface into the cell nucleus through inclusion of polypeptide domains for accomplishing receptor binding and internalization, as well as sequential endosomal escape and nuclear translocation. The objective of this study was to develop a new MNT targeted at folate receptors (FRs) for precise delivery of therapeutic cargo to the nuclei of FR-positive cells and to evaluate its potential, particularly for delivery of therapeutic agents (eg, the Auger electron emitter ${ }^{111} \mathrm{In}$ ) into the nuclei of target cancer cells.

Methods: A FR-targeted MNT was developed by site-specific derivatization of ligand-free MNT with maleimide-polyethylene glycol-folic acid. The ability of FR-targeted MNT to accumulate in target FR-expressing cells was evaluated using flow cytometry, and intracellular localization of this MNT was assessed using confocal laser scanning microscopy of cells. The cytotoxicity of the ${ }^{111}$ In-labeled FR-targeted MNT was evaluated on HeLa and U87MG cancer cell lines expressing FR. In vivo micro-single-photon emission computed tomography/CT imaging and antitumor efficacy studies were performed with intratumoral injection of ${ }^{111}$ In-labeled FR-targeted MNT in HeLa xenograft-bearing mice.

Results: The resulting FR-targeted MNT accumulated in FR-positive HeLa cancer cell lines specifically and demonstrated the ability to reach its target destination - the cell nuclei. ${ }^{111}$ In-labeled FR-targeted MNT demonstrated efficient and specific FR-positive cancer cell eradication. A HeLa xenograft in vivo model revealed prolonged retention of ${ }^{111}$ In delivered by FR-targeted MNT and significant tumor growth delay (up to $80 \%$ growth inhibition).

Conclusion: The FR-targeted MNT met expectations of its ability to deliver active cargo into the nuclei of target FR-positive cells efficiently and specifically. As a result of this finding the new FR-targeted MNT approach warrants broad evaluation.

Keywords: nuclear delivery, folic acid, cancer, radionuclide therapy, indium-111

\section{Introduction}

Therapeutic systems that are being developed to target abnormal (eg, cancer) cells with any therapeutic agent need high lesion-selectivity properties to "find" the specified cells while efficiently sparing normal cells. One promising approach to provide this is the use of lesion-specific surface cell receptors or antigens as a target for therapeutics. ${ }^{1}$ Folate receptors (FRs), which are frequently overexpressed and accessible to bloodcirculating agents either upon malignant transformation of the cell or upon activation of macrophages in contrast to their negligible exposure to the blood pool in normal tissues and non-activated macrophages, are among the best known and recognized types of these target antigens, ${ }^{1-4}$ FRs are prospective surface receptors for targeting
Correspondence: Alexander S Sobolev Laboratory of Molecular Genetics of Intracellular Transport, Institute of Gene Biology, 34/5 Vavilov St, II 9334 , Moscow, Russia

Tel +7499 I 353100

$\mathrm{Fax}+74991354105$

Email alsobolev@yandex.ru 
numerous diseases, mainly several types of cancer and a broad group of significant inflammatory conditions including, but not limited to, rheumatoid arthritis, ${ }^{5,6}$ atherosclerosis, ${ }^{7}$ Crohn's disease, ${ }^{8}$ and some other conditions.

There is a cohort of therapeutic agents (DNA; Auger electron emitters and alpha emitters producing short-range high linear energy transfer [LET] ionizing particles; photosensitizers, producing mainly reactive oxygen species, etc.) requiring (or at least highly "desiring") not only targeting to the specified abnormal cells but also further delivery into the subcellular target compartment, where their maximal therapeutic efficacy can be achieved..$^{9,10}$ To be more specific, we will consider Auger electron emitters as an example: Auger electrons emitted by most widely recognized Auger electron emitters $\left({ }^{125} \mathrm{I},{ }^{123} \mathrm{I},{ }^{67} \mathrm{Ga},{ }^{111} \mathrm{In}\right)$ used in clinical practice are characterized by tissue path lengths of $<50 \mathrm{~nm}$ (Figure 1A), which makes their cell-killing efficacy critically dependent on the exact site of decay inside the cell. ${ }^{11}$ When the site of decay is shifted from the cell surface to its nucleus, energy deposition in radiation-sensitive DNA has been calculated to increase up to 30-fold (Figure 1A). ${ }^{12}$ Moreover, cytotoxicity could increase by more than 100-fold when the decay site is located within close proximity of nuclear DNA. ${ }^{13}$ A prospective way to implement targeting at the subcellular
A

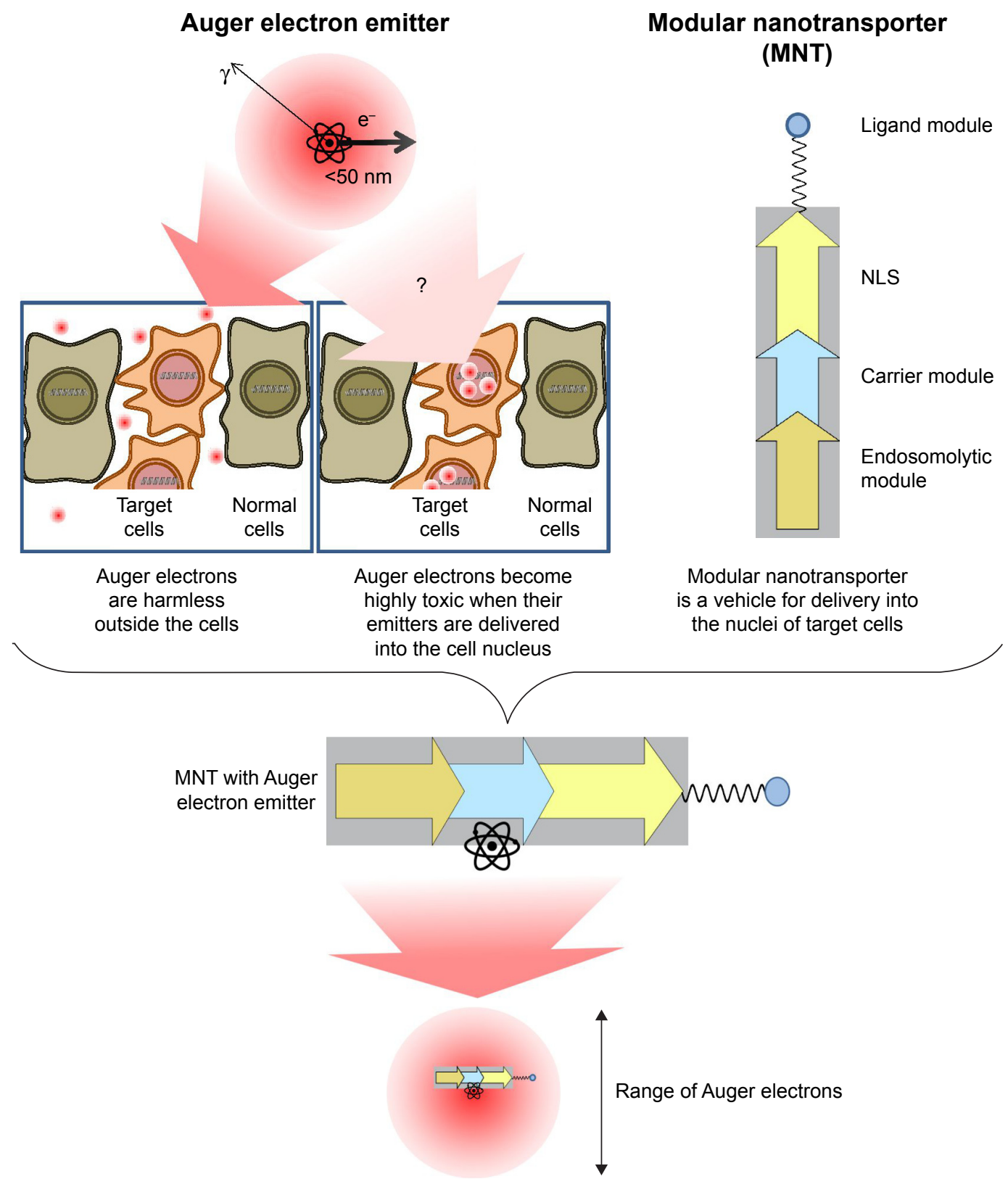

Figure I (Continued) 
B

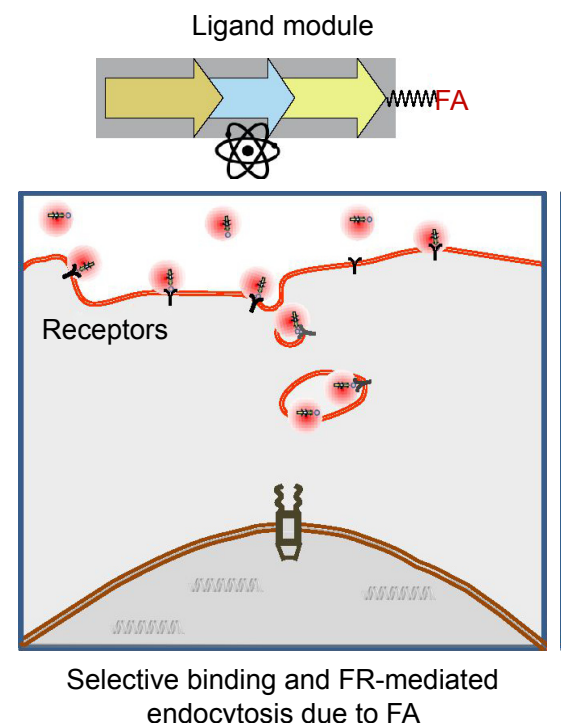

\section{Functions of MNT modules}
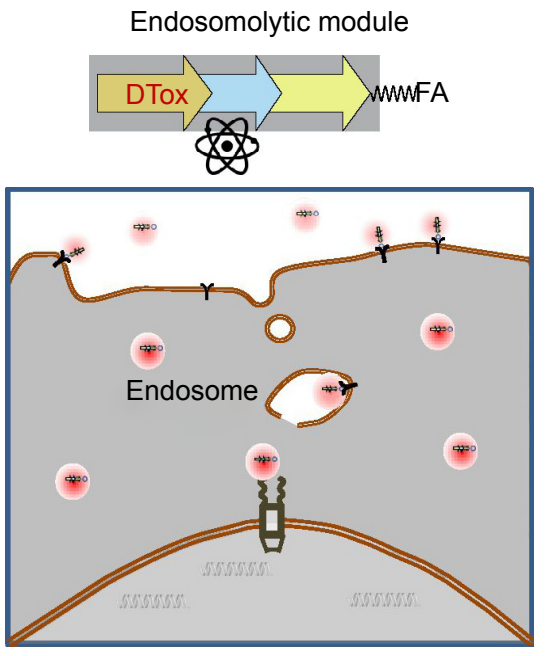

Endosome escape due to DTox
NLS

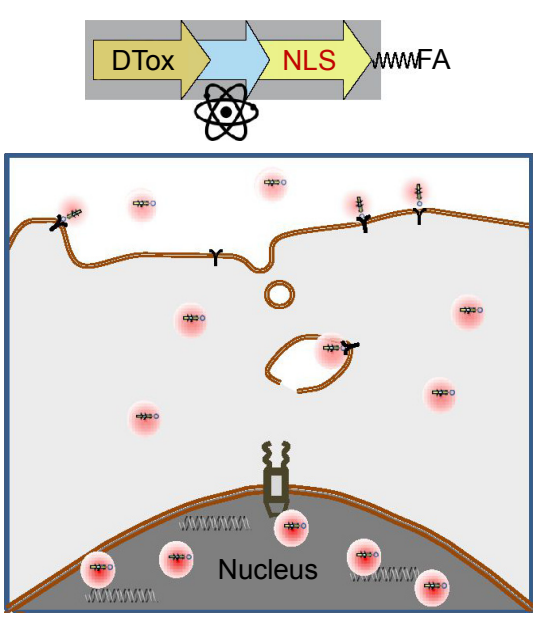

Transport into the nucleus due to NLS

Figure I Schematic presentation of the targeting delivery approach using targeted intranuclear delivery of cytotoxic drug (here, Auger electron emitter I'In) by folate receptor-targeted MNT.

Notes: (A) Auger electron range and the efficiency of Auger electron emitters located inside and outside the cell. (B) MNT module functions.

Abbreviations: DTox, diphtheria toxin translocation domain; FA, folic acid; MNTs, modular nanotransporters; NLS, nuclear localization sequence; PEG, polyethylene glycol.

level is to utilize natural intracellular transport pathways. ${ }^{9,10}$ Figure 1 demonstrates the benefits of this targeting delivery approach. A promising drug delivery system recruiting this strategy is the modular nanotransporters (MNTs) approach, recombinant molecules consisting of domains (artificial modules) for accomplishing receptor binding and internalization, as well as endosomal escape and nuclear translocation, thereby facilitating the delivery of drugs from the cell surface to the nucleus (Figure 1B). ${ }^{14}$ These "smart" vehicles targeting either epidermal growth factor receptor (EGFR) or melanocortin-1 receptor-overexpressing cancer cells have already proven their potential utility for delivering various therapeutic agents, including photosensitizers ${ }^{15-17}$ and radionuclides emitting high LET particles, ${ }^{18-20}$ as they were demonstrated to localize in the nuclei of target cancer cells in vitro (up to $60 \%$ internalized found in nuclei) $)^{15,16,18,20}$ and in vivo, ${ }^{17}$ as well as significantly enhance the cytotoxicity of these abovementioned cargoes in vitro ${ }^{15,16,18,20}$ and therapeutic potential of different photosensitizers ${ }^{17}$ and Auger electron emitter ${ }^{111} \mathrm{In}^{21}$ in vivo. Moreover, manifold advantage of full-size MNT over MNTs lacking any functional modules for delivery of cytotoxic drugs has been demonstrated previously. ${ }^{15,16}$

As the interchangeable nature of the modules within MNT offers the use of nearly any targeting ligand, an MNT concept combined with FR targeting seems to be a promising new approach for the treatment of numerous diseases characterized by FR overexpression.
The objective of the current study was to develop a new effective MNT specific to FR for targeted delivery of therapeutic cargo to the nuclei of FR-positive cells and to evaluate its potential for use of therapeutic agents. The Auger electron emitter ${ }^{111}$ In was used as an example due to its promising therapeutic potential and clinical acceptance for diagnostics. The principal scheme of the development of FR-targeted MNT and its labeling with ${ }^{111} \mathrm{In}$ is presented in Figure 2.

\section{Material and methods \\ Cell lines}

Human cervical carcinoma HeLa cells were kindly provided by Prof E Sverdlov, Institute of Bioorganic Chemistry, Russian Academy of Sciences, Moscow, Russia. Human glioma U87MG cells were kindly provided by Dr Darell Bigner, Duke University Medical Center, Durham, NC, USA. The use of these cell lines was approved by the Moscow State University Commission on Bioethics. The cells were cultured in in-house folate-deficient media supplemented with both $10 \%$ calf fetal serum (CFS) and gentamicin $(50 \mathrm{mg} / \mathrm{L}$, Dalhimpharm, Khabarovsk, Russia) at $37^{\circ} \mathrm{C}$ in a $5 \% \mathrm{CO}_{2}$ atmosphere.

\section{Folate receptor-targeted modular nanotransporter (MNT)}

FR-targeted MNT was made by attaching either folic acid (FA) polyethylene glycol (PEG) maleimide (FA-PEG-Mal, NanoCs, New York, NY, USA) or free FA (Sigma-Aldrich, 


\section{A}

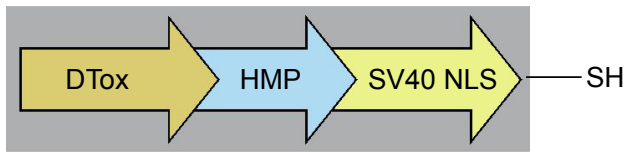<smiles>NC1=NC2C(=NC=C(Nc3ccc(C(=O)NC(CCC(=O)NCCCCN4C(=O)C=CC4=O)C(=O)O)cc3)N1)N=C(N)C2O</smiles>

Maleimide-PEG-N-folate

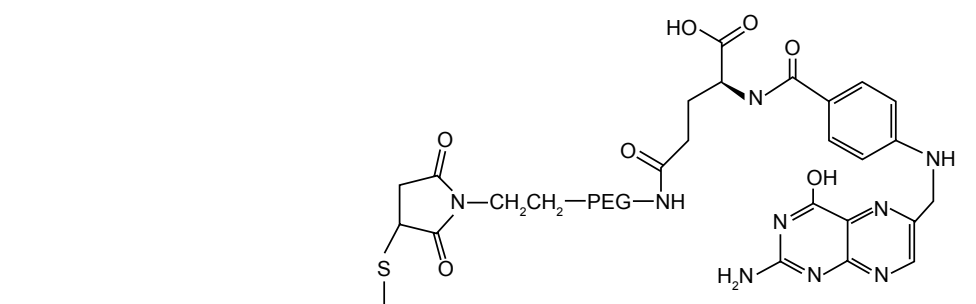

MNT-PEG-FA

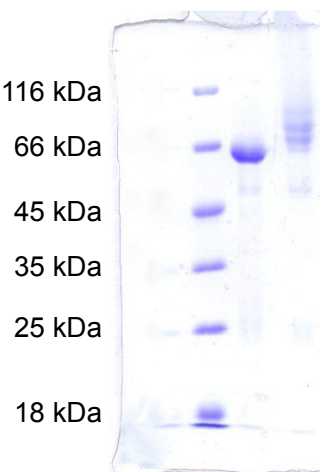

Standards MNT MNT-PEG-FA

B

MNT-PEG-FA

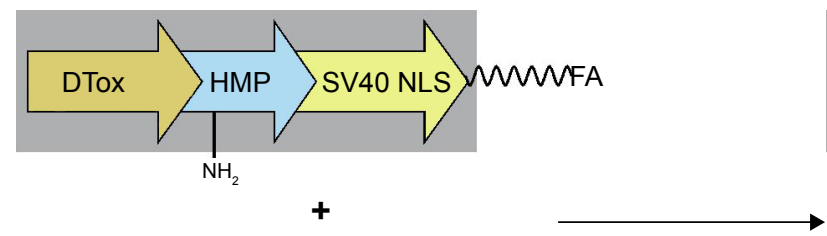

\section{p-SCN-Bn-NOTA}

\section{NOTA-MNT-PEG-FA}

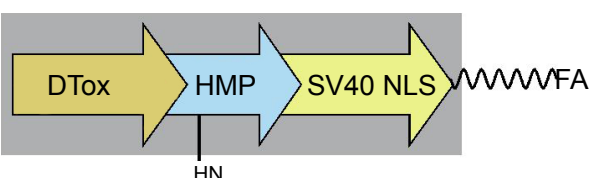

HN

$\mathrm{s}_{\mathrm{NH}}$

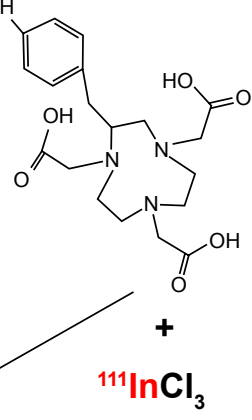

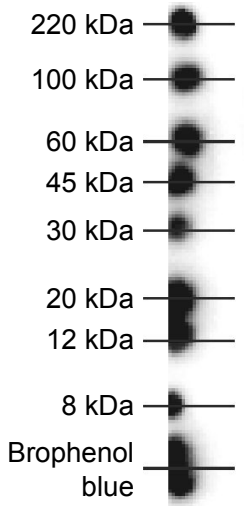

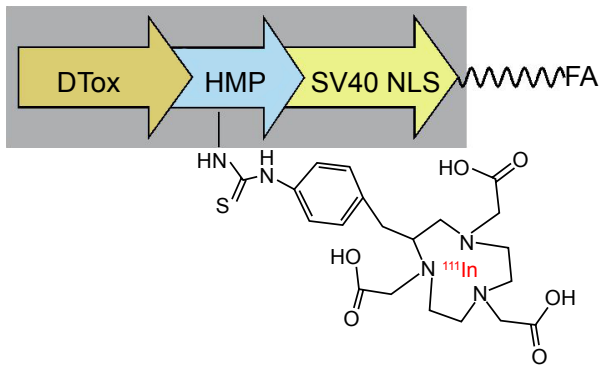

111In-MNT-PEG-FA

Figure 2 Development of FR-targeted MNT (MNT-PEG-FA).

Notes: (A) Schematic presentation of MNT derivatization with FA moiety with a SDS-PAGE gel under nonreducing conditions of MNT and the resulting FR-targeted MNT-PEG-FA. (B) Schematic presentation of MNT-PEG-FA labeling with Auger electron emitter "'In with autoradiography of SDS-PAGE gels of "'In-MNT-PEG-FA. Labels on the left indicate the bands from ColorBurst ${ }^{\mathrm{TM}}$ Electrophoresis Markers.

Abbreviations: DTox, diphtheria toxin translocation domain; FA, folic acid; HMP, hemoglobin-like protein of E. coli; MNTs, modular nanotransporters; NLS, nuclear localization sequence; PEG, polyethylene glycol; SDS-PAGE, sodium dodecyl sulfate polyacrylamide gel electrophoresis. 
St Louis, MO, USA) to a polypeptide consisting of the diphtheria toxin translocation domain as an endosomolytic module, the optimized nuclear localization sequence of SV-40 large T-antigen, and hemoglobin-like protein of Escherichia coli HMP as a carrier module; however, lacking any ligand module prepared as reported earlier. ${ }^{17}$ In brief, ligand-free MNT was expressed in E. coli suspension (optical density $\left.[\mathrm{OD}]_{550}: 0.6-1.0\right)$ by addition of isopropyl- $\beta-\mathrm{D}$ 1-thiogalactopyranoside to a final concentration of $200 \mu \mathrm{M}$ at $+18^{\circ} \mathrm{C}$ and shaking of $170-180 \mathrm{rpm}$ for $2 \mathrm{~h}$. The bacterial cells were pelleted by centrifugation at $10,000 \mathrm{rpm}$ in a JA-10 rotor (Beckman, Brea, CA, USA) at $+4^{\circ} \mathrm{C}$ for $30 \mathrm{~min}$, then the pellets were frozen for subsequent lysis in ice-cold $50 \mathrm{mM}$ sodium phosphate, $300 \mathrm{mM} \mathrm{NaCl}$, pH 8.0, $1 \mathrm{mM}$ phenylmethylsulfonyl fluoride, $5 \mathrm{mg} / \mathrm{mL}$ lysozyme, and $0.5 \%$ Triton $\mathrm{X}-100$. The resulting lysate was clarified by centrifugation at $18,000 \mathrm{rpm}$ in a JA-20 rotor (Beckman) at $+4^{\circ} \mathrm{C}$ for $30 \mathrm{~min}$, then the supernatant was loaded onto a Ni-NTA agarose column (QIAGEN, Venlo, the Netherlands), washed with $50 \mathrm{mM}$ sodium phosphate, $300 \mathrm{mM} \mathrm{NaCl}, 20 \mathrm{mM}$ imidazole, $\mathrm{pH} 8.0$, $0.5 \%$ Triton X-100, $1 \%$ glycerol, followed by $50 \mathrm{mM}$ sodium phosphate, $300 \mathrm{mM} \mathrm{NaCl}, 20 \mathrm{mM}$ imidazole, $\mathrm{pH}$ 8.0. The MNTs were eluted with $50 \mathrm{mM}$ sodium phosphate, $300 \mathrm{mM}$ $\mathrm{NaCl}, 700 \mathrm{mM}$ imidazole, $\mathrm{pH} 8.0$, and dialyzed against $10 \mathrm{mM}$ sodium phosphate, $150 \mathrm{mM} \mathrm{NaCl}, \mathrm{pH} 7.4$.

The first variant of FR-targeted MNT-FA was synthesized by chemical attachment of FA to ligand-free MNT according to a protocol described previously for FA attachment to albumin. ${ }^{22}$ Briefly, FA (Sigma-Aldrich, St. Louis, MO) was dissolved in dimethyl sulfoxide to $0.1 \mathrm{M}$ and incubated with a 1.2-fold molar excess of 1-ethyl-3-(3-dimethylaminopropyl) carbodiimide (EDC) for $1 \mathrm{~h}$ at room temperature (RT), followed by the addition of N-hydroxysuccinimide (NHS) in 1.2-fold molar excess. After $4 \mathrm{~h}$ incubation at RT, 15-fold molar excess of the "activated" FA was added to the ligandfree MNT solution and incubated for $12 \mathrm{~h}$ at RT. The unconjugated FA as well as reaction byproducts were removed by five cycles of ultrafiltration on Amicon Ultra Centrifugal Filter Units (Ultracel-30K, Merck Millipore Ltd, Tullagreen, Ireland), yielding purified MNT-FA. The FA:MNT ratio in the resulting MNT-FA was estimated spectrophotometrically (see details below).

To obtain the second FR-targeted MNT variant (Figure 2) requiring site-specific modification with $3.4 \mathrm{kDa}$ FA-PEGMal, we searched for optimal conditions of this reaction giving reasonable modification of MNT with FR-targeting ligand. The variables included: the sulfhydryl (SH)-reducing agent used (tris(2-carboxyethyl)phosphine hydrochloride
[TCEP] or 1,4-dithiothreitol [DTT]); TCEP concentration, and FA-PEG-Mal/MNT ratio in the reaction mixture. In our very first step of optimization of the conjugation protocol, we used model $\mathrm{NH}_{2}$-PEG-Mal instead of FA-PEG-Mal. The optimized protocol for MNT labeling that follows was used for subsequent experiments. In a typical procedure, freshly prepared $100 \mathrm{mM}$ TCEP solution ( $\mathrm{pH} 7.0$ ) was added to MNT solution in phosphate-buffered saline (PBS; $2.5 \mathrm{mg} / \mathrm{mL}$, $33 \mu \mathrm{M}$ ) to a final concentration of TCEP of $3 \mathrm{mM}$. This mixture was incubated for $40 \mathrm{~min}$ at $37^{\circ} \mathrm{C}$ to reduce $\mathrm{SH}$-groups within the MNT molecule (three SH-groups in total). The mixture was dialyzed against PBS with $1 \mathrm{mM}$ ethylenediaminetetraacetic acid (EDTA; pH 7.3) to reduce TCEP concentration to $8.2 \mu \mathrm{M}$; then freshly prepared FA-PEG-Mal solution in PBS with EDTA was added to reduced MNT in 50-fold molar excess to MNT. The reaction mixture was incubated overnight at $+4^{\circ} \mathrm{C}$ with gentle mixing. The resulting MNT with FA-PEG-Mal (MNT-PEG-FA) was purified from unreacted FA-PEG-Mal as well as reaction byproducts by five cycles of ultrafiltration using Amicon Ultracel-30K. The yield of the FA-PEG-Mal conjugation reaction (the percentage of MNT molecules modified with FA-PEG-Mal) was calculated by two different methods. The first was performed by analysis of nonreducing sodium dodecyl sulfate polyacrylamide gel electrophoresis (SDS-PAGE) using ImageQuant TL 5.0 software (Bio-Rad, Hercules, CA, USA), calculating the percentage of bands corresponding to intact MNT $(70 \mathrm{kDa})$ and MNT derivatized with one $(73.5 \mathrm{kDa})$, two $(77 \mathrm{kDa})$, or three $(80.5 \mathrm{kDa})$ Mal-PEG-FA. The second method utilized spectrophotometric determination. In detail, the absorbance of Mal-PEG-FA conjugated MNT was determined at $\lambda=367 \mathrm{~nm}$, and the concentration of FA was calculated using the Beer-Lambert law and the previously determined extinction coefficient of FA in carbonate buffer with $5 \mathrm{mM}$ EDTA, pH 8.6, in which the probes were diluted for spectroscopy $\left(\varepsilon_{367 \mathrm{~nm}}=11,000 \mathrm{M}^{-1} \mathrm{~cm}^{-1}\right)$, corrected for MNT absorbance at $\lambda=367 \mathrm{~nm}$. The concentration of protein (MNT) was determined using the Bradford protein assay.

Control MNT-PEG lacking FA ligand was prepared in the same way using Mal-PEG-amine (Mw $5 \mathrm{kDa}$; Creative PEGWorks, Chapel Hill, NC, USA).

\section{Conjugation of Alexa Fluor 647 to MNT-PEG-FA and MNT-PEG}

Freshly prepared solution of Alexa Fluor 647 succinimidyl ester (Molecular Probes, Eugene, OR, USA) ( $2 \mathrm{mg} / \mathrm{mL}$ ) was added to either MNT-PEG-FA or MNT-PEG solution $(2.5 \mathrm{mg} / \mathrm{mL})$ in carbonate buffer, $\mathrm{pH}$ 8.6, in 5:1 molar excess. 
The reaction mixture was incubated overnight at $+4^{\circ} \mathrm{C}$ with gentle mixing. The resulting MNT-PEG-FA-Alexa647 or MNT-PEG-Alexa647 was purified from unreacted Alexa647 as well as reaction byproducts by five cycles of ultrafiltration using Amicon Ultracel-30K. The average amount of Alexa Fluor 647 molecules conjugated to MNT-PEG-FA or MNT-PEG molecules was assessed spectrophotometrically. In detail, the absorbance of MNTs conjugated with Alexa Fluor 647 was determined at $\lambda=650 \mathrm{~nm}$, and the concentration of Alexa Fluor 647 was calculated using the BeerLambert law and the extinction coefficient of Alexa Fluor $647 \varepsilon_{650 \mathrm{~nm}}=270,000 \mathrm{M}^{-1} \mathrm{~cm}^{-1}$. The concentration of protein (MNTs) was determined using the Bradford protein assay.

\section{Flow cytometry studies of MNT-PEG-FA-} Alexa647 internalization in FR-positive cells HeLa (FR-positive) or A549 (ATCC ${ }^{\circledR}$ CCL-185 ${ }^{\mathrm{TM}}$, FR-negative) cells were seeded in 24-well plates (4-9×10 4 cells/well). The next day, the medium was changed for fresh medium containing $1 \%$ bovine serum albumin (BSA). Subsequently, MNT-PEG-FA-Alexa647 or MNTPEG-Alexa647 were added to the wells (three to six per each point) to a final concentration of $50 \mathrm{nM}$, and the cells were incubated for 24 or $48 \mathrm{~h}$ at $37^{\circ} \mathrm{C}$ in a $5 \% \mathrm{CO}_{2}$ humidified atmosphere. Afterward, medium containing unbound MNTs was removed and the cells were washed thrice, trypsinized, harvested into Eppendorf tubes, and centrifuged at $200 \mathrm{rpm}$ for $10 \mathrm{~min}$. The supernatant containing MNT-PEG-FAAlexa647 or MNT-PEG-Alexa647 detached by trypsin from the cell surface was discarded, and the remaining cell pellet containing only internalized fraction of MNT-PEGFA-Alexa647 was dissolved in Hanks solution with 1\% BSA and analyzed by flow cytometry using an Epics Altra Flow Cytometer (Beckman Coulter, Miami, FL, USA). The Alexa Fluor 647 dye was excited at $633 \mathrm{~nm}$ and emission was detected at $675 \mathrm{~nm}$. A total of $0.5-1 \times 10^{4}$ gated events were collected per sample.

To assess the nonspecific uptake, parallel wells (three to six per each point) with FA-containing media ( $1 \mathrm{mM})$ were processed in the same way. To determine cell autofluorescence, the addition of MNT-PEG-FA-Alexa647 or MNTPEG-Alexa647 was omitted and the wells were processed in the same way.

\section{Confocal laser scanning microscopy imaging of FR-positive live cells after incubation with MNT-PEG-FA-Alexa647}

HeLa (FR-positive) or A549 (FR-negative) cells were seeded on coverslips in 24 -well plates $\left(4-9 \times 10^{4}\right.$ cells/well).
The next day, the medium was changed to fresh medium containing 1\% BSA. Subsequently, MNT-PEG-FA-Alexa647 or MNT-PEG-Alexa647 was added to the wells to the final concentration of $50 \mathrm{nM}$, and the cells were incubated for $48 \mathrm{~h}$ at $37^{\circ} \mathrm{C}$ in a $5 \% \mathrm{CO}_{2}$ humidified atmosphere. Afterward, the medium containing unbound MNTs was removed, and the cells were washed four times with Hanks solution followed by acetone-methanol $(1: 1)$ fixation for $30 \mathrm{~min}$ at $-40^{\circ} \mathrm{C}$. Then the cells were air-dried, and the plate was closed tightly and stored at $-40^{\circ} \mathrm{C}$ until imaging. Prior to imaging, the cells were permeabilized in PBS with $0.1 \%$ Tween-20 and $0.2 \%$ Triton $\mathrm{X}-100$ for $35 \mathrm{~min}$ at $37^{\circ} \mathrm{C}$, then stained with 4',6-diamidino-2-phenylindole (DAPI) (Sigma) and mounted on a glass microscope slide using Shandon Immumount mounting medium (Thermo Scientific, Pittsburgh, PA, USA). Subcellular localization was investigated using an LSM 510 META NLO multiphoton laser scanning microscope (Carl Zeiss, Oberkochen, Germany) equipped with a Mai Tai Broadband Mode-Locked Ti-sapphire Laser (Spectra-Physics, Mountain View, CA, USA) with PlanApochromat $\times 63 / 1.4$ Oil DIC lens. DAPI fluorescence was two-photon excited with a Ti-sapphire Laser (790 nm), while Alexa Fluor 647 fluorescence was registered at $633 \mathrm{~nm}$ excitation and 650-710 pass band for emission.

To assess nonspecific uptake, parallel wells with coverslips with FA-containing medium $(1 \mathrm{mM})$ were processed in the same way. To determine cell autofluorescence, the addition of MNT-PEG-FA-Alexa647 or MNT-PEG-Alexa647 was omitted, and the coverslips were processed in the same way.

\section{Labeling of MNT-PEG-FA and MNT-FA with "II In Conjugation of $\mathrm{p}-\mathrm{SCN}-\mathrm{Bn}-\mathrm{NOTA}$ to MNTs}

To permit subsequent ${ }^{111} \mathrm{In}$ labeling, the chelator $\mathrm{p}-\mathrm{SCN}-\mathrm{Bn}$ NOTA (Macrocyclics, Plano, TX, USA) $\left(\log \mathrm{K}_{\mathrm{In} 3+\mathrm{NOTA}}=26.2\right)^{23}$ was conjugated to MNT-PEG-FA and MNT-FA according to a recently published protocol. ${ }^{18}$ Briefly, $1 \mathrm{mg}$ of MNT-FA or 4-8 mg of MNT-PEG-FA was incubated with 10 -fold molar excess of the chelator in $1 \times \mathrm{pH} 8.6$ conjugation buffer ${ }^{18}$ for $20 \mathrm{~h}$ at RT with final concentrations of $\mathrm{MNT} \geq 1.5 \mathrm{mg} / \mathrm{mL}$. The chelator-MNT conjugate was concentrated and separated from excess chelator by five cycles of ultrafiltration using Amicon Ultracel-30K. During this process, the conjugation buffer was gradually replaced with $10 \mathrm{mM}$ 4-(2-hydroxyethyl)-1-piperazineethanesulfonic acid (HEPES), $15 \mathrm{mM} \mathrm{NaCl}, \mathrm{pH}$ 7.4. All buffers used for chelator conjugation and labeling procedures were passed through Chelex-100 resin (200-400 mesh; Bio-Rad) to minimize adventitious metal ion contamination. 


\section{Labeling of MNT Conjugates with "'In}

NOTA-MNT-PEG-FA or NOTA-MNT-FA $(0.12 \mathrm{mg})$ in $10 \mathrm{mM}$ HEPES, $15 \mathrm{mM} \mathrm{NaCl}$, pH 7.5, was mixed with $29 \mu \mathrm{L}$ 1 M HEPES, pH 7.5, $21 \mu \mathrm{L} 0.1 \mathrm{M}$ citrate, $\mathrm{pH} 6.7,8 \mu \mathrm{L} \mathrm{1 \%}$ SDS, and $42 \mu \mathrm{L} 0.25 \mathrm{M} \mathrm{HCl}$ (Ultrapure Grade, Merck, Darmstadt, Germany); then $102 \mu \mathrm{L}(322 \mathrm{MBq}){ }^{111} \mathrm{InCl}_{3}$ (Federal Center of Nuclear Medicine Projects Design and Development, Moscow, Russia) in $0.048 \mathrm{M} \mathrm{HCl}$ was added. The reaction mixture was incubated at $37^{\circ} \mathrm{C}$ for $1 \mathrm{~h}$, and then the reaction was stopped by adding $3 \mu \mathrm{L} 0.05 \mathrm{M}$ EDTA, $\mathrm{pH} 8.0$, followed by gentle mixing and incubation for $10 \mathrm{~min}$ at $37^{\circ} \mathrm{C}$. Finally, the $\mathrm{pH}$ was neutralized with $24 \mu \mathrm{L} 1 \mathrm{M} \mathrm{NaOH}$. The initial specific radioactivity of either FR-targeted ${ }^{111} \mathrm{In}$-MNT, obtained using this protocol, was $2.7 \mathrm{GBq} / \mathrm{mg}$. As a control, ${ }^{111}$ In was treated following the same procedures except that the NOTA-MNT was omitted in the reaction mixture. Radiochemical yields and ${ }^{111} \mathrm{In}$-MNT-PEG-FA and ${ }^{111} \mathrm{In}$ MNT-FA integrity were analyzed by Laemmli SDS-PAGE using Any $\mathrm{kD}^{\mathrm{TM}}$ Mini-PROTEAN ${ }^{\circledR}$ TGX $^{\mathrm{TM}}$ Precast Protein Gels (Bio-Rad) with subsequent detection of radioactivity on a Storm 865 phosphorimager (GE Healthcare, Uppsala, Sweden). The images were analyzed using ImageQuant TL 5.0 software (Bio-Rad). Due to high $(96+\%)$ radiochemical labeling yields, there was no need for any subsequent purification of ${ }^{111}$ In-labeled MNTs.

\section{Cytotoxicity studies}

HeLa and U87MG cells were seeded in 24-well plates $\left(2.5 \times 10^{4}\right.$ cells $/$ well $)$. Two days later, the medium was refreshed and dilutions of ${ }^{111} \mathrm{In}-\mathrm{MNT}-\mathrm{PEG}-\mathrm{FA}(0-25 \mathrm{MBq} / \mathrm{mL}$; $0-11 \mu \mathrm{g} / \mathrm{mL})$, or ${ }^{111} \mathrm{In}-\mathrm{MNT}-\mathrm{FA}(0-20 \mathrm{MBq} / \mathrm{mL})$, or the control ${ }^{111} \mathrm{In}$ solution containing $(0-25 \mathrm{MBq} / \mathrm{mL})$, or nonlabeled NOTA-MNT-PEG-FA $(0-11 \mu \mathrm{g} / \mathrm{mL})$ was added. For FA competition studies, $500 \mu \mathrm{M}$ FA was added to the HeLa cells prior to the addition of ${ }^{111}$ In-MNT-PEG-FA or control ${ }^{111} \mathrm{In}$. The cells were incubated for $48 \mathrm{~h}$ in a humidified atmosphere at $37^{\circ} \mathrm{C}$ in $5 \% \mathrm{CO}_{2}$. Then medium containing unbound radioactivity was removed, and the cells were washed, trypsinized, harvested, and resuspended in $1 \mathrm{~mL}$ of fresh medium. The cells were seeded for colony-forming assay in $25-\mathrm{cm}^{2}$ flasks (2,000 cells per flask) in DMEM/F12 supplemented with 10\% CFS. After 6-11 days, the colonies were stained with Crystal Violet and counted.

\section{Animal studies}

These experiments were performed on $8-10$-week-old female Balb/c nu/nu mice (Institute of Bioorganic Chemistry Regional Affiliate, Russian Academy of Science, Pushchino, Russia). The animals were maintained under specific pathogen-free conditions with access to mouse chow and water ad libitum. Starting from 10 days prior to single-photon emission computed tomography (SPECT)/CT imaging or therapy and up to 2 weeks after initiation of the experiment, the mice were fed with FA-deficient diet TD.00434 (Envigo, Huntingdon, UK). The experimental protocol was approved by the Institute Commission for Animals and was performed in accordance with the National Institutes of Health guide for the care and use of laboratory animals. HeLa tumors were established in nude mice by subcutaneous injection of $10^{7}$ cells suspended in $100 \mu \mathrm{L}$ serum-free medium into the back flank region. SPECT/CT imaging and therapy studies were initiated 12 days after tumor inoculation.

\section{SPECT/CT imaging}

For SPECT/CT imaging, HeLa xenograft-bearing mice were anesthetized with $0.8 \%-1.8 \%$ isoflurane in air; tumor-bearing mice $(n=3)$ received intratumoral injections of $15 \mathrm{MBq}$ of either ${ }^{111} \mathrm{In}$-MNT-PEG-FA or control ${ }^{111} \mathrm{In}$ in Hanks solution in a volume equal to half of the tumor volume. Whole-body imaging was performed on a U-SPECT-II/CT (MILabs, Utrecht, the Netherlands) scanner immediately after injection and continued for $5 \times 10$-min frames using a 1.0-mm-diameter pinhole collimator, with subsequent immediate whole-animal CT acquisition. Additional SPECT/ $\mathrm{CT}$ imaging was performed during the subsequent days (3-5 frames $\times 10 \mathrm{~min}$ ). The images were reconstructed using U-SPECT-Rec2.34b software obtained from the manufacturer, followed by co-registration of SPECT images to the corresponding CT images. Quantitative analysis of images after three dimensional (3D)-reconstruction was performed using PMOD 3.4 software (PMOD Technologies Ltd., Zurich, Switzerland).

The radioactivity concentration in tumor and other organs or tissues was determined by selecting a few spheres within the organ or tissue of interest, followed by division of the summarized signal within this sphere by its volume.

\section{Therapy studies}

For therapy studies, HeLa xenograft-bearing mice $(n=4-5)$ were intratumorally injected with $7.5 \mathrm{MBq} / 3.5 \mu \mathrm{g}\left({ }^{111} \mathrm{In}-\right.$ MNT-PEG-FA only), or $15 \mathrm{MBq} / 7.1 \mu \mathrm{g}$ of either ${ }^{111}$ In-MNTPEG-FA, or control ${ }^{111}$ In or unlabeled NOTA-MNT-PEG-FA in a volume equal to half of the tumor volume.

The efficacy of the treatment was monitored using the tumor growth inhibition inde ${ }^{24}$ defined as: $(1-$ [mean volume of treated tumors $] /[$ mean volume of control tumors $]) \times 100 \%$.

The animals were euthanized when the tumor volume exceeded $2,000 \mathrm{~mm}^{3}$ or at the sign of animal distress. 


\section{Statistics}

The data were analyzed using GraphPad Prism 5 software (GraphPad Software Inc., San Diego, CA, USA). Data on the plots represent mean values, with bars indicating the standard error of the mean of 3-6 repetitive values. The significance of the difference was evaluated using the Kruskal-Wallis or F-test or log-rank test.

\section{Results}

\section{Folate receptor-targeted MNT}

Ligand-free MNT was expressed and purified successfully to $90+\%$ purity (Figure $2 \mathrm{~A}$ ). For our first steps, we conjugated FA to MNT directly via NHS and EDC according to a previously published protocol, ${ }^{22}$ yielding MNT-FA with FA:MNT molar ratio of 1.6, which was determined spectrophotometrically by absorbance at $367 \mathrm{~nm}$ with subsequent correction for protein absorbance at this wavelength. Then, to improve the characteristics of the final product, we switched to site-specific attachment of FA-PEG-Mal compound to SH-groups of MNT. An optimal part of the MNT for attachment of additional functional groups (eg, low-molecular-weight drug) is its carrier module. This module contains two cysteines, which are, however, not readily available for modification. Therefore, to obtain better yield of Mal-PEG-FA attachment, we used a MNT variant with an additional cysteine residue in place of the ligand module on the C-terminus of the MNT, because ligand modules of other similar MNTs do not alter the functionality of other modules. ${ }^{15,16}$ Conjugation of Mal-PEG-FA to MNT required some efforts to find the optimal reaction conditions, giving MNT-PEG-FA with high reaction yield. These reaction optimization steps are presented in the Supplementary material and Figure S1.

The resulting MNT-PEG-FA was characterized by two different methods. Using the first one, nonreducing SDSPAGE, we calculated the ratio of MNT derivatized with one, two or three PEG-FA molecules and non-derivatized MNT as well as evaluated the integrity of the resulting MNT-PEG-FA. The resulting product of MNT modification with Mal-PEG-FA possessed predominantly MNT containing one PEG-FA molecule, though the presence of MNT molecules modified with two or even three PEG-FA (Figure 2A) points at partial availability of the two other $\mathrm{SH}$-groups under the reaction conditions.

The second method, spectrophotometry, was used to estimate the average number of FA molecules attached to MNT relying on FA characteristic absorbance peak at $367 \mathrm{~nm}$, corrected to MNT absorbance at this wavelength. Both spectrophotometric and SDS-PAGE gel analysis methods gave a similar FA-PEG:MNT ratio of 1.5-1.6.

\section{Conjugation of Alexa Fluor 647 to MNT-PEG-FA and MNT-PEG}

For subsequent flow cytometry and confocal laser scanning microscopy studies, we conjugated Alexa Fluor 647 succinimidyl ester to MNT-PEG-FA or MNT-PEG, resulting in an average of 3.5 Alexa Fluor 647 molecules attached to MNT-PEG-FA.

\section{Flow cytometry studies of MNT-PEG-FA- Alexa647 internalization in FR-positive cells}

Using flow cytometry studies on trypsinized and washed cells, we assessed only the internalized fraction of Alexa Fluor 647-labeled MNT-PEG-FA. We observed significant fluorescence of HeLa (FR-positive) cells incubated with MNT-PEG-FA-Alexa647 (Figure 3). The median fluorescence intensity (MFI) increased with increase in incubation time from 24 to $48 \mathrm{~h}$ (Figure 3 ). For further comparisons, we set MFI values of HeLa cells incubated with MNT-PEGFA-Alexa647 to $100 \%$ for each time point. The MFI was significantly reduced to $9.9 \%$ at $24 \mathrm{~h}$ and to $14 \%$ at $48 \mathrm{~h}$ using medium containing free $1 \mathrm{mM} \mathrm{FA}$, thus demonstrating specificity of MNT-PEG-FA-Alexa647 internalization (Figure 3). The MFI of HeLa cells incubated with ligand-free control MNT-PEG-Alexa647 was reduced to $6.6 \%$ at $24 \mathrm{~h}$ and to $8.4 \%$ at $48 \mathrm{~h}$ compared to HeLa cells incubated with MNT-PEG-FA-Alexa647, showing that conjugation of FA enhances the uptake of MNT-PEG-FA by tumor cells. In contrast to MNT-PEG-FA-Alexa647, there was no difference in the MFI of HeLa cells incubated with ligand-free control MNT-PEG-Alexa647 in the presence or absence of $1 \mathrm{mM}$ FA, demonstrating nonspecific internalization of control MNT-PEG-Alexa647 lacking FA. Moreover, FR-negative A549 cells demonstrated more than 10-fold reduced MFI, which was similar for both MNT-PEG-FA-Alexa647 and control ligand-free MNT-PEG-Alexa647.

\section{Confocal laser scanning microscopy imaging of FR-positive cells after incubation with MNT-PEG-FA-Alexa647}

Confocal laser scanning microscopy studies performed on the fixed FR-expressing HeLa cells (Figure 4) demonstrates intracellular and intranuclear Alexa Fluor 647 fluorescence after $48 \mathrm{~h}$ incubation with MNT-PEG-FA-Alexa647 (Figure 4A and $\mathrm{A}^{\prime}$ ). As MNT internalization and transport to nuclei via the cytoplasm is going on from outside continuously, we can observe a "snapshot" of intracellular distribution of the MNT, with a significant part caught on its way (eg, in the cytoplasm) 


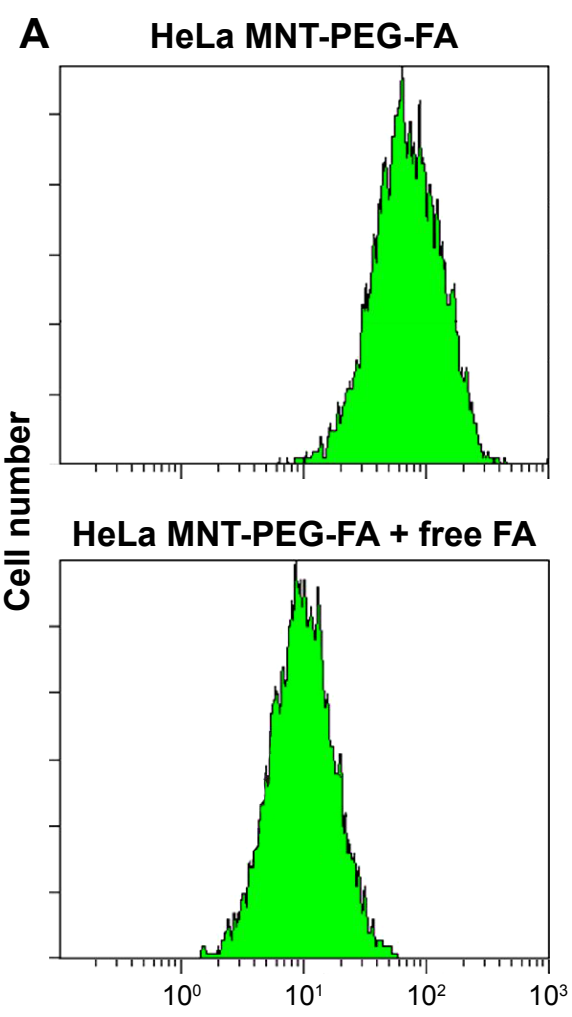

B

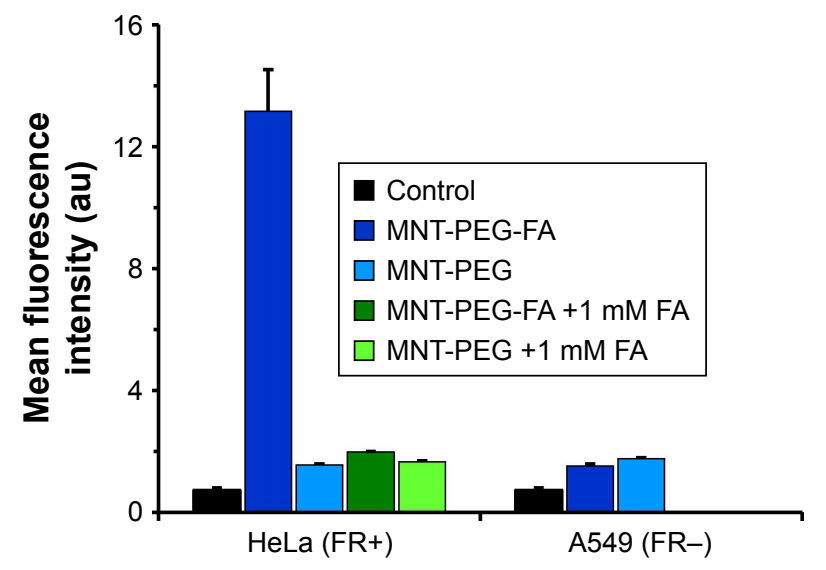

HeLa MNT-PEG

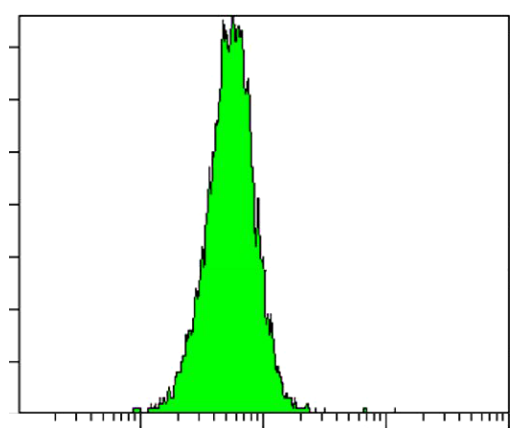

HeLa MNT-PEG + free FA

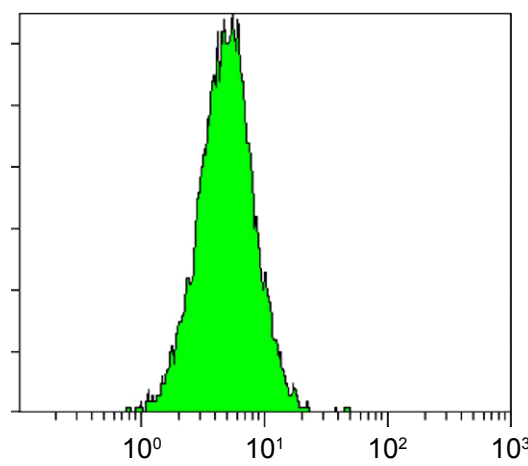

Fluorescence intensity (au)
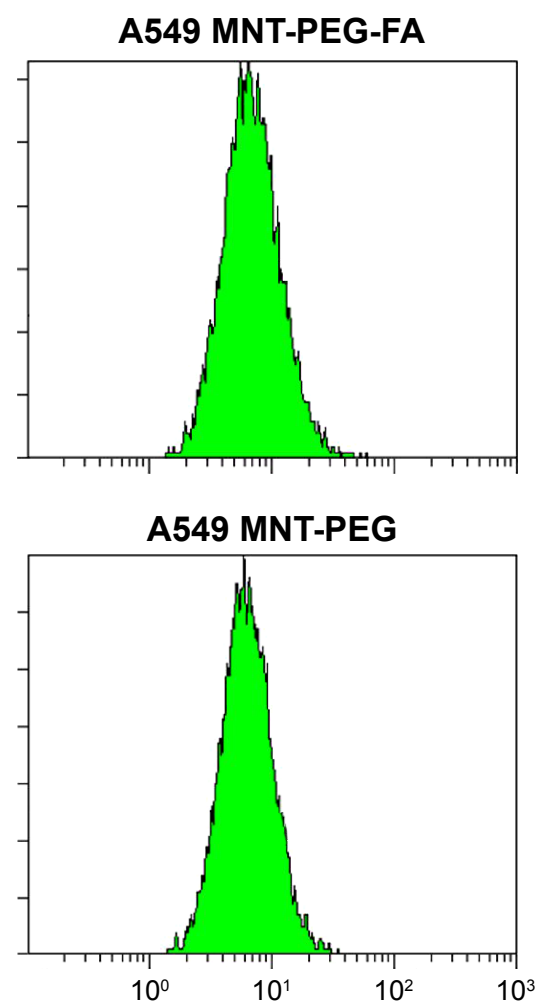

$48 \mathrm{~h}$

C

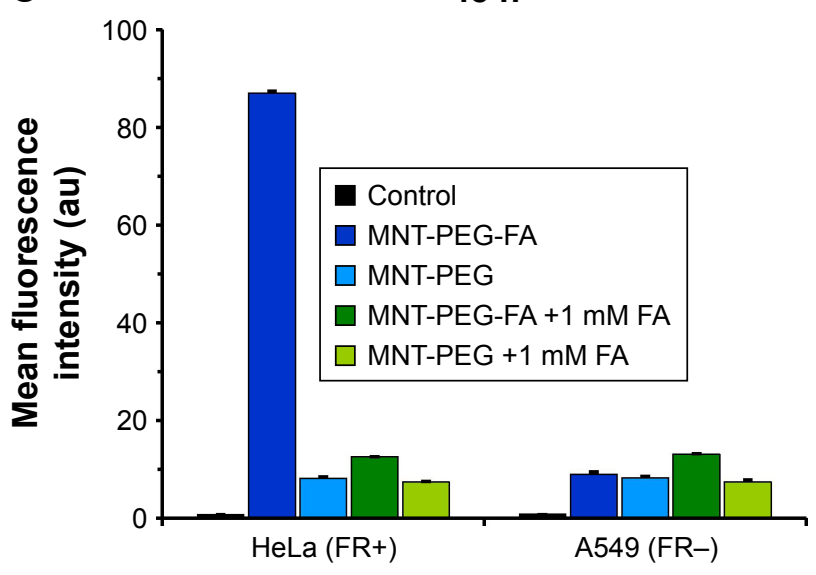

Figure 3 Intracellular accumulation of MNT-PEG-FA labeled with Alexa Fluor 647 and measured by flow cytometry.

Notes: (A) Flow cytometry histograms of HeLa (FR-positive) or A549 (FR-negative) cells incubated with either 50 nM MNT-PEG-FA-Alexa647 alone or in the presence of I mM FA or with control non-targeted MNT-PEG-Alexa647, lacking FA alone or in the presence I mM FA for 48 h. (B, C) Mean fluorescence intensities for HeLa or control A549 cells incubated with either $50 \mathrm{nM}$ MNT-PEG-FA-Alexa647 alone or in the presence of I mM FA or with control non-targeted MNT-PEG-Alexa647, lacking FA alone or in the presence I mM FA for $24 \mathrm{~h}$ (B) or $48 \mathrm{~h}(\mathbf{C})$. Untreated cells served as autofluorescence controls. Error bars represent standard errors of mean ( $\mathrm{n}=3-6)$.

Abbreviations: FA, folic acid; MNTs, modular nanotransporters; PEG, polyethylene glycol.

to the target compartment - the cell nucleus. The presence of free FA in the medium, as well as the use of non-targeted control MNT-PEG-Alexa647 reduced the signal dramatically (Figure 4B, B', D, and D'). FR-negative A549 cells demonstrated barely visible signal, which did not depend on the presence of free FA in the medium or the use of non-targeted control MNT-PEG (Figure 4G, G', H, H', I, and I').

\section{Labeling of MNT-PEG-FA and MNT-FA with "'In}

Both MNT-FA and MNT-PEG-FA were successfully conjugated with chelator NOTA, and subsequently, the resulting conjugates were efficiently labeled with Auger electron emitter ${ }^{111}$ In with specific radioactivity of $2.7 \mathrm{GBq} / \mathrm{mg}$. According to SDS-PAGE data, the ${ }^{111}$ In labeling procedure 


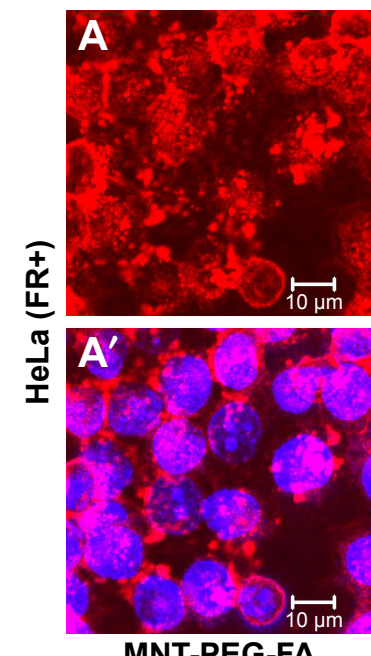

MNT-PEG-FA
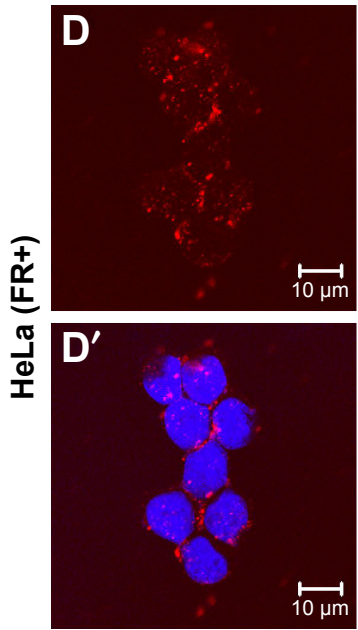

MNT-PEG

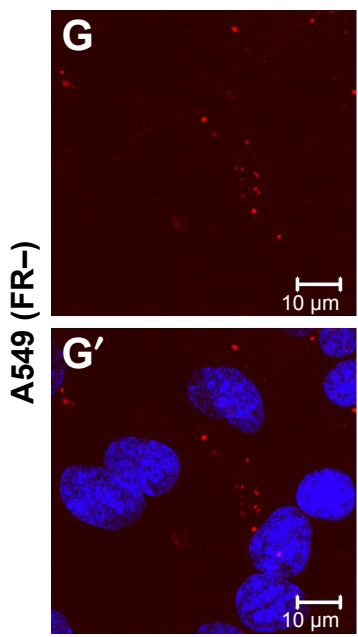

MNT-PEG-FA
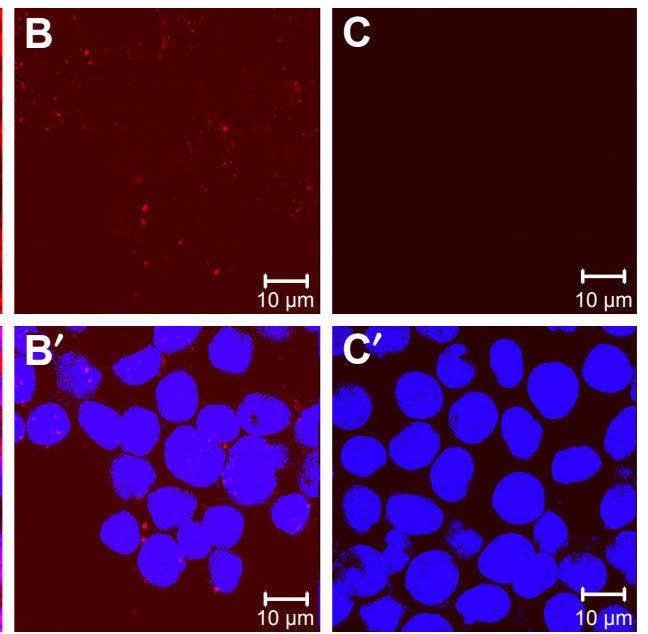

MNT-PEG-FA + free $F A$
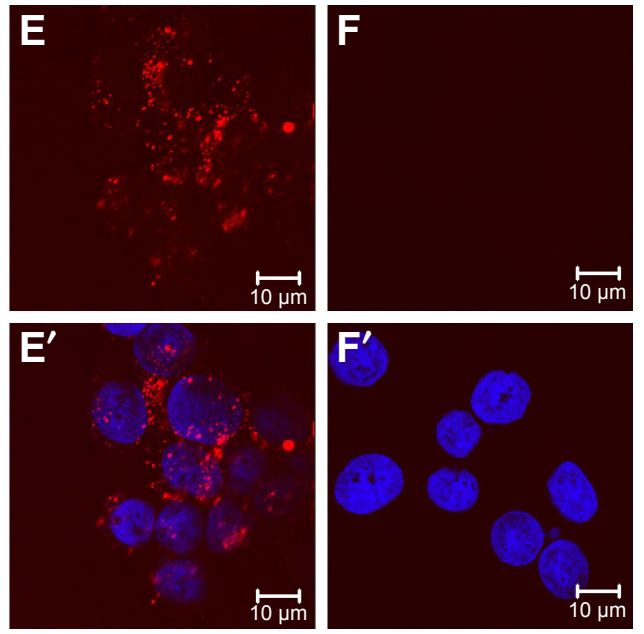

MNT-PEG + free FA

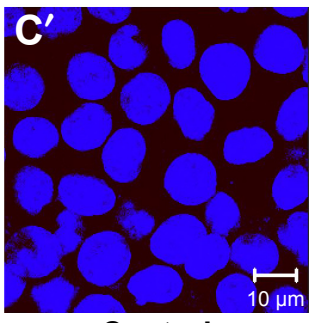

Control
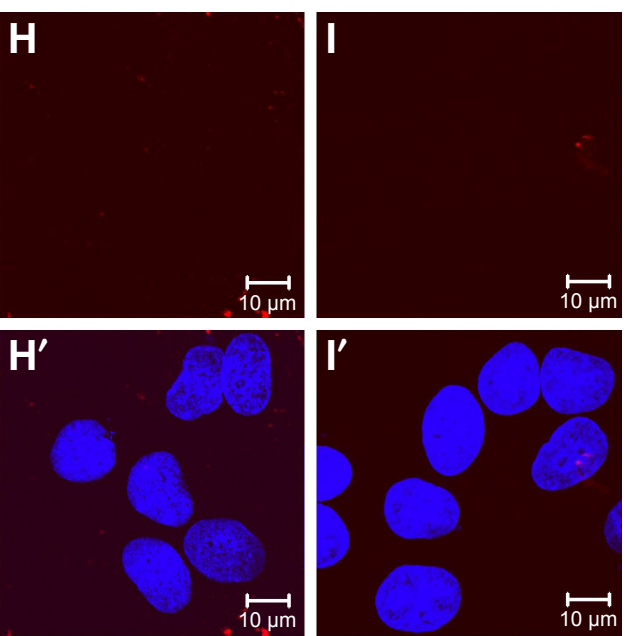

MNT-PEG + free FA

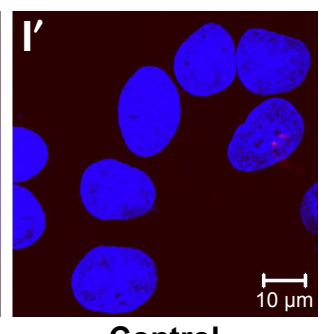

Control

Figure 4 Intracellular distribution of MNT-PEG-FA labeled with Alexa Fluor 647 and visualized by confocal laser scanning microscopy.

Notes: Confocal laser scanning microscopy images (slices made across the nuclei) of HeLa (FR-positive) or A549 (FR-negative) cells incubated with either 50 nM MNT-PEG-FA-Alexa647 alone (A, $\left.\mathbf{A}^{\prime}, \mathbf{G}, \mathbf{G}^{\prime}\right)$ or in the presence I mM FA (B, B', H, H') or with control non-targeted MNT-PEG-Alexa647, lacking FA alone or in the presence ImM FA for $48 \mathrm{~h}$. Untreated cells served autofluorescence controls; Alexa Fluor 647 fluorescence corresponding to MNT-PEG-FA or MNT-PEG is in red pseudocolor $(\mathbf{A}-\mathbf{C}, \mathbf{D}-\mathbf{F}, \mathbf{G}-\mathbf{I})$, and overlay of Alexa Fluor 647 fluorescence and DAPI staining for nuclei is presented in blue pseudo-color $\left(\mathbf{A}^{\prime}-\mathbf{C}^{\prime}, \mathbf{D}^{\prime}-\mathbf{F}^{\prime}, \mathbf{G}^{\prime}-\mathbf{I}^{\prime}\right)$. Bar $=10 \mu \mathrm{m}$. Plan-Apochromat $\times 63 / 1.4$ Oil DIC lens, zoom $2 \times$.

Abbreviations: FA, folic acid; MNTs, modular nanotransporters; PEG, polyethylene glycol. 
did not alter the integrity of the resulting ${ }^{111}$ In-MNT-PEG-FA (Figure 2B). The stability of ${ }^{111}$ In-MNT-FA was evaluated by serial SDS-PAGE over 8 days of incubation in serum at $37^{\circ} \mathrm{C}$, and typical results are shown in Figure S2. The results indicated excellent retention of label on the MNT under these conditions: after 7 days of incubation in serum, $<3.5 \%$ of ${ }^{111}$ In was released from ${ }^{111}$ In-MNT-FA.

The labeling yields were close to quantitative $(97.3 \% \pm 0.4 \%$ [n=3] for ${ }^{111}$ In-MNT-FA and $96.3 \% \pm 1.2 \%$ [n=6] for
${ }^{111}$ In-MNT-PEG-FA); thus, there was no need for any subsequent purification of the ${ }^{111}$ In-labeled MNTs.

\section{In vitro cytotoxic efficiency}

The clonogenic survival plots of HeLa cells after 48-h incubation with serial dilutions of ${ }^{111}$ In-MNT-PEG-FA $(0-25 \mathrm{MBq} / \mathrm{mL})$ or ${ }^{111} \mathrm{In}-\mathrm{MNT}-\mathrm{FA}(0-20 \mathrm{MBq} / \mathrm{mL})$ are given in Figure 5A, demonstrating the benefit of site-specific attachment of the FA moiety with a PEG spacer over direct FA attachment to the MNT.
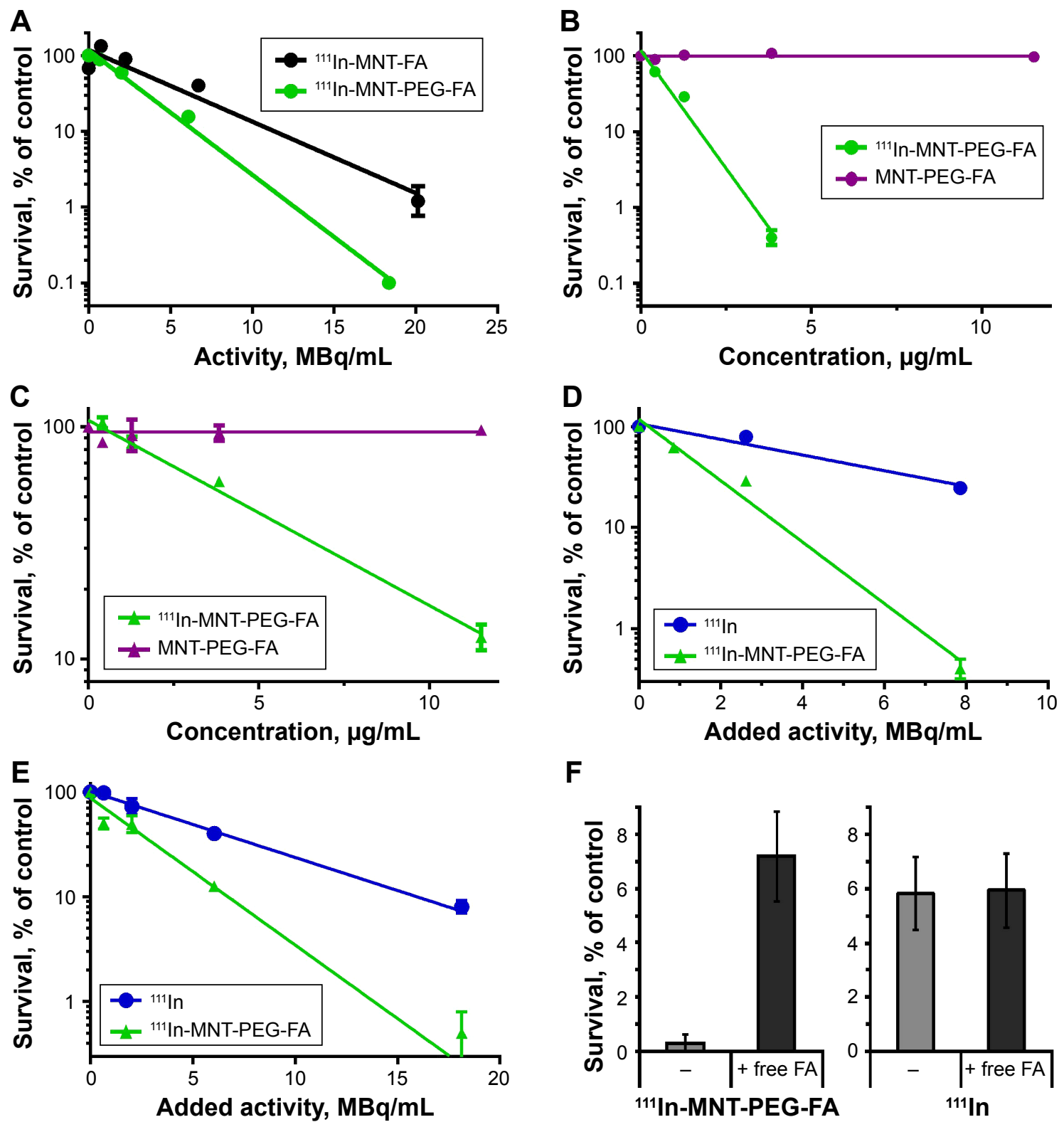

$\mathbf{F}$

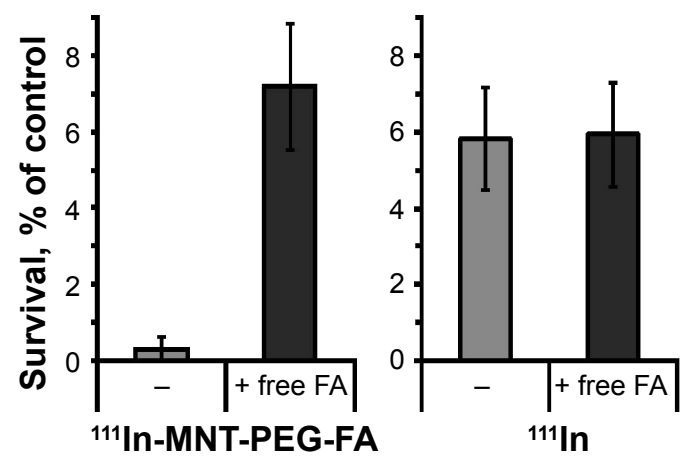

Figure 5 Cytotoxic efficiency of "'In delivered by FR-targeted MNTs.

Notes: (A) Cytotoxicity of "'In-MNT-FA on HeLa cells compared to "''In-MNT-PEG-FA. (B, C) Cytotoxicity of "'In-MNT-PEG-FA compared to unlabeled MNT-PEG-FA on HeLa (B) and U87MG (C) cells; (D, E) Cytotoxicity of "'In-MNT-PEG-FA compared to control "'In on HeLa (D) and U87MG (E) cells. (F) Cytotoxicity of "''In-MNTPEG-FA and control '"'In in the presence or absence of $500 \mu \mathrm{M}$ free FA on HeLa cells. The solid lines represent fits of the data to a mono-exponential model. Error bars represent standard errors of mean $(n=3-6)$.

Abbreviations: FA, folic acid; MNTs, modular nanotransporters; PEG, polyethylene glycol. 
The clonogenic survival plots of FR-positive U87MG and HeLa cells after a 48-h incubation with serial dilutions of ${ }^{111} \mathrm{In}-\mathrm{MNT}-\mathrm{PEG}-\mathrm{FA}(0-11 \mu \mathrm{g} / \mathrm{mL})$ or non-labeled MNT-PEG-FA $(0-11 \mu \mathrm{g} / \mathrm{mL})$ are given in Figure 5B and $\mathrm{C}$. These results demonstrate that within the studied concentration range, unlabeled MNT-PEG-FA itself is completely nontoxic.

The cytotoxic effect of ${ }^{111}$ In delivered by MNT-PEG-FA was enhanced dramatically compared to control ${ }^{111} \mathrm{In}$ (Figure 5D and E).

Moreover, cytotoxic effectiveness of ${ }^{111}$ In delivered by MNT-PEG-FA was significantly reduced in the presence of free FA, while no influence of free FA on ${ }^{111}$ In cytotoxicity was observed (Figure 5F), indicating the specificity of

${ }^{111}$ In-MNT-PEG-FA efficiency.

\section{Animal studies \\ SPECT/CT imaging}

Following intratumoral injection, ${ }^{111}$ In-MNT-PEG-FA demonstrated good intratumoral retention of ${ }^{111}$ In activity (Figure 6A-C). The decay-corrected retention half-life from the HeLa xenograft was calculated to be $\sim 52 \mathrm{~h}$. (Figure $6 \mathrm{~B}$ ). Detection of radioactivity following intratumoral administration of ${ }^{111}$ In-MNT-PEG-FA in normal tissues was low (Figure 6A and C), mostly limited to some detectable signal in the kidneys and liver. In contrast to ${ }^{111} \mathrm{In}$-MNT-PEG-FA control, ${ }^{111}$ In was rapidly eliminated from the tumor (the decaycorrected retention half-life was $\sim 24 \mathrm{~min}$ ) (Figure S3).

\section{Antitumor efficacy}

The potential of MNT-PEG-FA as a strategy for FRtargeted delivery of therapeutic cargo was investigated after
A

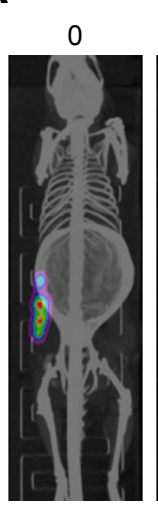

Min
Time, days after injection

1
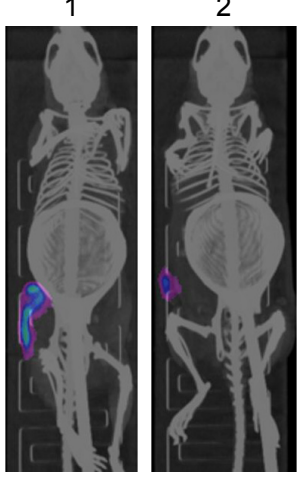

3

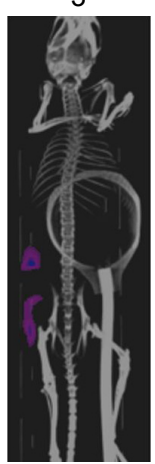

5

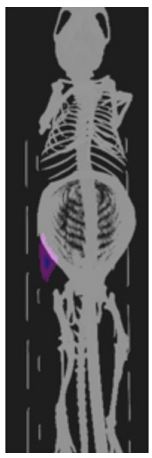

Max
B

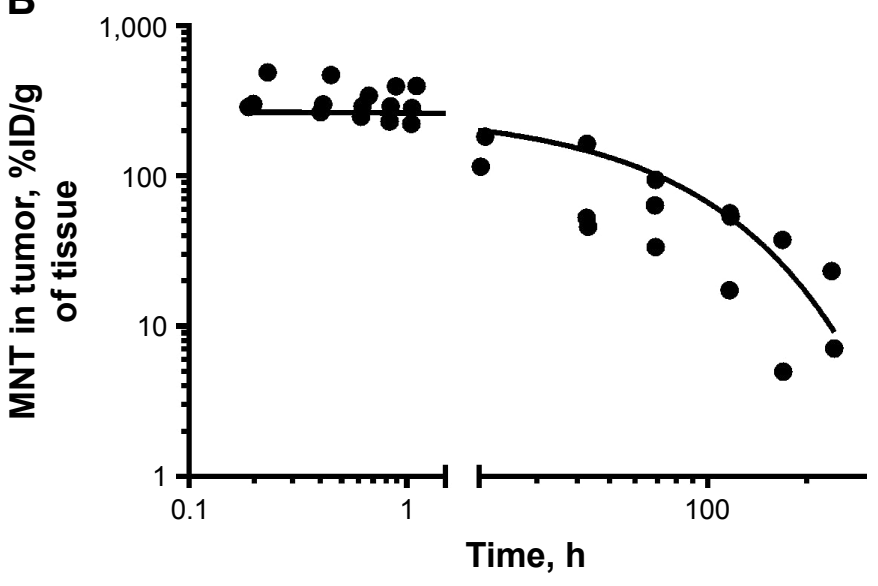

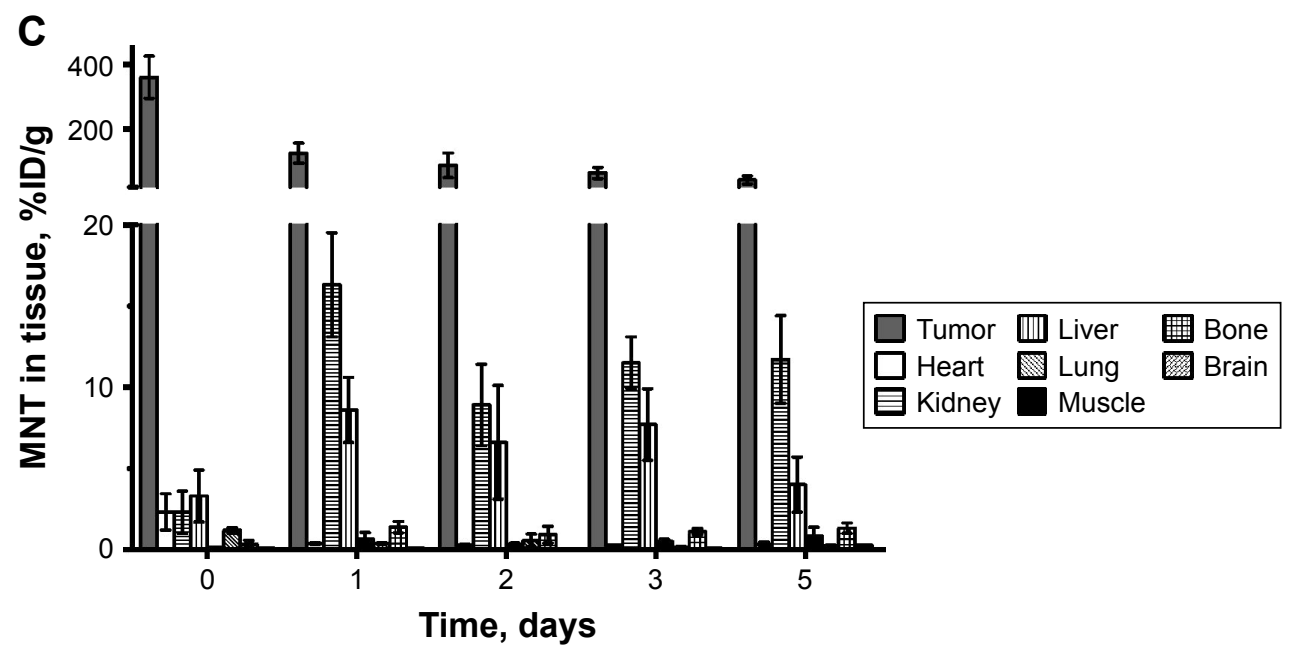

Figure 6 Serial SPECT/CT imaging of nude mice bearing HeLa human cervical carcinoma xenografts after intratumoral injection of '"'In-MNT-PEG-FA.

Notes: (A) SPECT/CT (color/gray) images of a representative animal at indicated times after injection of "'In-MNT-PEG-FA. (B) Decay-corrected retention of I'In activity in tumor following "'In-MNT-PEG-FA injection. The solid line represents fit of the data to a monoexponential model. The ring in the abdominal region presented on the CT scans of the mice is the breathing sensor. (C) Tumor retention and normal tissue redistribution of decay-corrected "'In activity with time.

Abbreviations: FA, folic acid; MNTs, modular nanotransporters; PEG, polyethylene glycol; SPECT, single-photon emission computed tomography. 
A

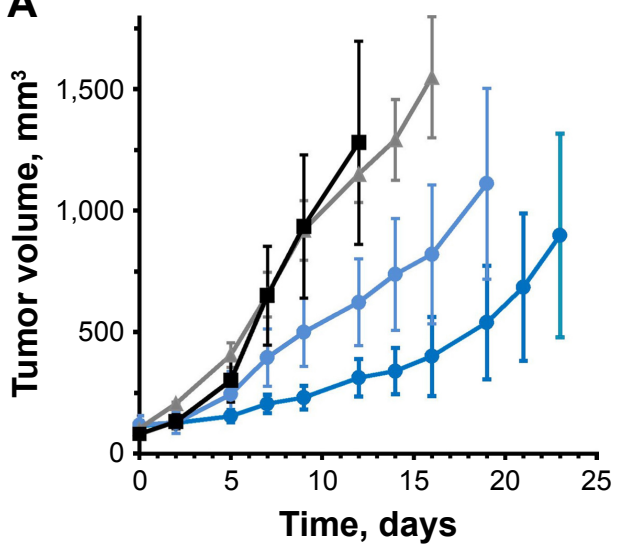

$\multimap{ }^{111}$ In-MNT-PEG-FA, $15 \mathrm{MBq}$

$\multimap{ }^{111}$ In-MNT-PEG-FA, $7.5 \mathrm{MBq}$

$\simeq$ Control ${ }^{111} \mathrm{In}, 15 \mathrm{MBq}$

$\rightarrow$ MNT without ${ }^{111} \mathrm{In}$
B

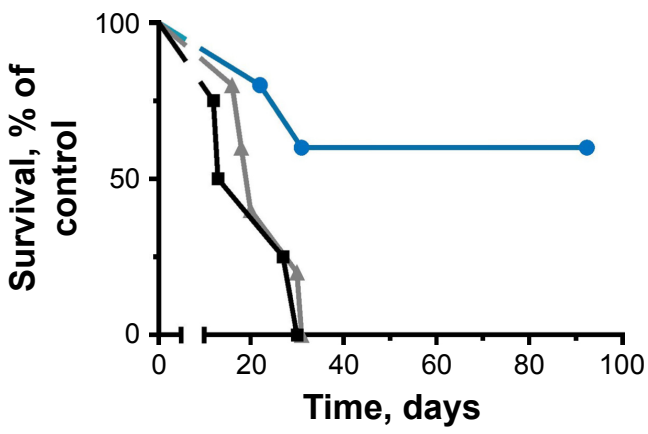

Figure 7 In vivo therapy of HeLa human cervical carcinoma-bearing mice with "'In-MNT-PEG-FA.

Notes: (A) Tumor growth kinetics of HeLa-bearing mice following a single intratumoral injection of "'In-MNT-PEG-FA (I5 MBq/7.I $\mu g$ and 7.5 MBq/3.5 $\mu$ g), control II'In (I5 MBq), or unlabeled MNT-PEG-FA (7.I $\mu \mathrm{g})$. (B) Survival curves for the selected treatment groups.

Abbreviations: FA, folic acid; MNTs, modular nanotransporters; PEG, polyethylene glycol.

intratumoral injection in mice with subcutaneous HeLa xenografts. Dose-dependent tumor growth delay was observed in the experiments where ${ }^{111} \operatorname{In}(7.5$ and $15 \mathrm{MBq}$ per mouse) was delivered by MNT-PEG-FA (Figure 7A). The higher dose was more efficient, demonstrating up to $80 \%$ tumor growth inhibition index compared to animals receiving a corresponding dose of non-labeled MNT. The corresponding dosage of control ${ }^{111} \mathrm{In}$ did not result in any tumor growth inhibition. The survival curve for the group of mice receiving $15 \mathrm{MBq}$ of ${ }^{111}$ In-MNT-PEG-FA differed significantly from the survival curves of mice in the control groups that received either $15 \mathrm{MBq}$ of ${ }^{111}$ In or non-labeled MNT-PEG-FA $(P=0.0289$, long-rank test) (Figure 7B). No significant weight loss was observed in any treatment group (Figure S4).

\section{Discussion}

Due to their accessibility to blood-borne agents and being confined almost exclusively to abnormal (eg, malignant) cells, FR is an attractive target for systems delivering either imaging agents or therapeutics or both to the site of disease. Therefore, delivery systems aimed at FR have been developed during recent decades, with a few of them recently undergoing or currently undergoing Phase I-III clinical trials with promising results. This includes mainly FA conjugated either to cytotoxic or imaging agents: Vinca alkaloid alone (ClinicalTrials.gov identifiers: NCT00308269; NCT01577654), ${ }^{25}$ and its combination with mitomycin ${ }^{26}$ as a therapeutic approach; indium-111 (ClinicalTrials.gov Identifier: NCT00003763 ${ }^{27}$ and technecium-99m (ClinicalTrials. gov Identifier: NCT01689766) ${ }^{28}$ for subsequent SPECT imaging; fluorescent infrared dye (ClinicalTrials.gov Identifier: NCT02317705) $)^{29}$ for intraoperative imaging, and some other variants. Besides these molecules, there are a large number of delivery systems targeted at FR currently in in vitro/preclinical stages of development, mainly FAdecorated liposomes, ${ }^{30}$ micelles, ${ }^{31}$ and nanoparticles ${ }^{32}$ demonstrating promising potency, which shows the perspectives of FR-targeted therapeutics and diagnostics approaches. They usually deliver the carried cargo into cells via FR-mediated endocytosis with subsequent cargo entrapment or active release $^{33}$ from the lysosomes, sometimes followed by passive transport to an intracellular compartment mediated by the properties of the cargo (eg, doxorubicin accumulating in the nucleus due to its binding to DNA). ${ }^{33}$

Aside from that, there is a cohort of prospective therapeutic/theranostic agents requiring active precise delivery to the subcellular target, usually into the cell nucleus, to be effective in terms of therapy. ${ }^{10}$ To accomplish this, we have developed a concept of MNTs providing specific transport of the attached therapeutic agent from the surface to the target pathological cell nucleus. This concept realized previously in melanocortin receptors-1 (MC1R) and EGFR-targeted MNTs proved its feasibility and high potency in vitro ${ }^{15-17,19,20}$ and in vivo, ${ }^{17,21}$ with each module retaining functionality and 
asserting the necessity of its inclusion within the MNT. ${ }^{15,16}$ The goal of this study was to develop a new targeted system combining FR-targeting strategy with the MNT approach that provides specific transport of the attached therapeutic agent from the surface to the target pathological cell nucleus, where the delivered agent is the most potent.

The first step of our work was the production of a new MNT, which required its post-translational derivatization with FA representing the MNT's ligand module for FR binding and internalization. We started from "straightforward" modification of MNT with FA via NHS and EDC as a simple, facile and relatively efficient route. The resulting MNT-FA demonstrated an increase in cytotoxicity for ${ }^{111}$ In delivered by MNT-FA compared to control ${ }^{111} \mathrm{In} .{ }^{21}$ These results pointed at the feasibility of the FR-targeted MNT approach and led us to develop a more sophisticated variant with an FA-moiety attached site-specifically to the MNT, in order to avoid the potential pitfalls of direct FA conjugation, mainly the possibility of activating the amino group of FA, thus altering its binding ability ${ }^{34}$ and potentially altering the activity of functional modules within the MNT.

Based on the data of Lee and Low reporting $\sim 3 \mathrm{kDa}$ PEG as the optimal spacer for FA attachment to FR-targeted liposomes, ${ }^{35}$ we chose an FA moiety containing a $3 \mathrm{kDa}$ PEG spacer between FA and the maleimide group. This spacer is supposed to give additional freedom to the FA within the MNT, which can presumably ease its binding to FR on target cells.

For our in vitro and in vivo studies, we chose cells (HeLa and U87MG) characterized by low-to-moderate ${ }^{36,37}$ expression of FR to assess the potency of FR-targeted MNT for the average FR-expressing cancer cells. Even on these cells possessing moderate FR expression, we observed the ability of the resulting MNT-PEG-FA to internalize efficiently, with more than 10-fold inhibition in the presence of excess free FA, suggesting a receptor-mediated process (Figure 3 ).

Consistent with the mission of the MNT, the resulting MNT-PEG-FA demonstrated the ability to reach the target FR-positive cell nuclei, as demonstrated by confocal laser scanning microscopy (Figure 4). MNT-PEG-FA modification with NOTA necessary for the subsequent ${ }^{111}$ In labeling did not alter the transporter's ability to reach the target cell nuclei (Figure S5).

For further assessment of the potential utility of MNTPEG-FA in vitro and in vivo, we chose, as an example, one of the prospective therapeutic cargoes, Auger electron emitter ${ }^{111} \mathrm{In}$, whose efficiency is highly dependent on targeted precise intranuclear delivery. Though being widely recognized and accepted in clinic as an imaging agent, no therapeutic agent based on this promising radionuclide has yet been developed, mainly due to the necessity of its nuclear delivery for efficient cell killing.

MNT-PEG-FA met our expectations of efficacy (Figure 5B-E) and specificity (Figure 5F) when delivering ${ }^{111}$ In. Moreover, as anticipated, this MNT-PEG-FA, where FA is attached site-specifically, demonstrated improved efficacy when compared to MNT-FA with direct FA conjugation via NHS and EDC. Based on these encouraging results, we started with the evaluation of the principal feasibility of the FR-targeted MNT approach in vivo. For the in vivo proof of principle, we chose the locoregional administration route for our initial experiments to get rid of numerous factors influencing the drug on its way to the target in the blood circulation. The retention of ${ }^{111}$ In-MNT-PEG-FA within the tumor is similar to our previously published data obtained for another type of MNT targeted at MC1R. ${ }^{21}$ Compared to intratumorally injected ${ }^{188}$ Re-labeled anti-HER2 antibody Herceptin $(\sim 150 \mathrm{kDa}),{ }^{38}{ }^{111} \mathrm{In}$-labeled MNTs possess significantly prolonged tumor retention. Many factors can result in this superior retention of MNTs. First, the ${ }^{111} \mathrm{In}$-labeling method that was used for radiolabeling of both MC1R-targeted ${ }^{21}$ and FR-targeted MNTs results in a highly stable product $(<3.5 \%$ ${ }^{111}$ In released after 7-day incubation) compared to moderate stability of ${ }^{188}$ Re-labeled Herceptin $\left(>7 \%{ }^{111}\right.$ In released after 1-day incubation). ${ }^{38}$ Second, superior tumor retention of ${ }^{111} \mathrm{In}$ MNT-PEG-FA can be mediated by its in vitro demonstrated ability to internalize cells efficiently and reach the cell nuclei, thus escaping degradation fate in lysosomes. Third, Herceptin, being an antibody, can escape cells via transcytosis, a process that seems unlikely for MNT-PEG-FA.

The resulting FR-targeted MNT demonstrated similar potency as MSH-targeted MNT, ${ }^{21}$ providing tumor growth retardation reaching $80 \%$ compared to control unlabeled FR-targeted MNT. Together with the fact of broad FRoverexpression in various diseases, this new approach seems to be of potential value and warrants further evaluation of this strategy's potency. Based on the fact of negligible FR exposure to the blood pool in normal tissues, we plan to adapt the FR-targeted MNT strategy for systemic administration, which can be beneficial for the elimination of metastasis along with the primary tumor. However, the intratumoral route of administration giving high local concentration of the therapeutic agent and devoid of numerous problems and adverse effects associated with systemic route (rapid clearance, normal tissue toxicity, weak intratumoral penetration, elimination by Kupffer cells, etc. $)^{39-42}$ can present independent interest for the treatment of such malignant tumors as cervical cancer. ${ }^{43}$ 
In this case, prolonged retention labeled MNT-PEG-FA, along with its specific local effects on cancer cells, could be useful in the clinic.

\section{Conclusion}

Based on the attractiveness of FR as a target for lesion-specific therapeutics and high potency of the MNT approach for precise delivery of cargo into the nuclei of target cells, we developed FR-targeted MNT by post-translational site-specific derivatization with FA representing the MNT's ligand module for FR binding. The resulting FR-targeted MNT demonstrated the ability to reach its target destination - the cell nuclei of FR-expressing cells. When delivering a cytotoxic agent, ${ }^{111}$ In FR-targeted MNT demonstrated efficient and specific FR-positive cancer cell eradication, as well as encouraging therapeutic potential on HeLa xenograft in vivo model. Taken together, these data warrant broad evaluation of this new FR-targeted MNT approach for delivery of various active cargoes into the nuclei of target FR-expressing cells.

\section{Acknowledgments}

This work was supported by Russian Science Foundation Grant 14-14-00874. Experiments were carried out with the use of the equipment from MV Lomonosov Moscow State University Program of Development and the Center of Collective Usage of the Institute of Gene Biology.

\section{Author contributions}

TAS, AAR, and ASS designed and evaluated the study. TAS, AAR, YVK, TSK, and SAO performed the study and data analysis. TAS, AAR, and ASS wrote the manuscript. All authors read and approved the final manuscript. All authors contributed toward data analysis, drafting and critically revising the paper and agree to be accountable for all aspects of the work.

\section{Disclosure}

The authors report no conflicts of interest in this work.

\section{References}

1. Slastnikova TA, Rosenkranz AA, Zalutsky MR, Sobolev AS. Modular nanotransporters for targeted intracellular delivery of drugs: folate receptors as potential targets. Curr Pharm Des. 2015;21(9): 1227-1238.

2. Lu Y, Low PS. Folate-mediated delivery of macromolecular anticancer therapeutic agents. Adv Drug Deliv Rev. 2002;54(5):675-693.

3. Muller C, Schibli R. Prospects in folate receptor-targeted radionuclide therapy. Front Oncol. 2013;3:249.

4. Srinivasarao M, Galliford CV, Low PS. Principles in the design of ligand-targeted cancer therapeutics and imaging agents. Nat Rev Drug Discov. 2015;14(3):203-219.
5. Paulos CM, Turk MJ, Breur GJ, Low PS. Folate receptor-mediated targeting of therapeutic and imaging agents to activated macrophages in rheumatoid arthritis. Adv Drug Deliv Rev. 2004;56(8):1205-1217.

6. van der Heijden JW, Oerlemans R, Dijkmans BA, et al. Folate receptor beta as a potential delivery route for novel folate antagonists to macrophages in the synovial tissue of rheumatoid arthritis patients. Arthritis Rheum. 2009;60(1):12-21.

7. Furusho Y, Miyata M, Matsuyama T, et al. Novel therapy for atherosclerosis using recombinant immunotoxinagainst folatereceptor betaexpressing macrophages. J Am Heart Assoc. 2012;1(4):e003079.

8. Turk MJ, Breur GJ, Widmer WR, et al. Folate-targeted imaging of activated macrophages in rats with adjuvant-induced arthritis. Arthritis Rheum. 2002;46(7):1947-1955.

9. Rosenkranz AA, Ulasov AV, Slastnikova TA, Khramtsov YV, Sobolev AS. Use of intracellular transport processes for targeted drug delivery into a specified cellular compartment. Biochemistry (Mosc). 2014;79(9):928-946.

10. Sobolev AS, Aliev RA, Kalmykov SN. Radionuclides emitting shortrange particles and modular nanotransporters for their delivery to target cancer cells. Russian Chemical Reviews. 2016;85(9):1011-1032.

11. Cornelissen B, Vallis KA. Targeting the nucleus: an overview of Auger-electron radionuclide therapy. Curr Drug Discov Technol. 2010; 7(4):263-279.

12. Goddu SM, Howell RW, Rao DV. Cellular dosimetry: absorbed fractions for monoenergetic electron and alpha particle sources and S-values for radionuclides uniformly distributed in different cell compartments. J Nucl Med. 1994;35(2):303-316.

13. Buchegger F, Perillo-Adamer F, Dupertuis YM, Delaloye AB. Auger radiation targeted into DNA: a therapy perspective. Eur J Nucl Med Biol Mol Imaging. 2006;33(11):1352-1363.

14. Sobolev AS. Modular transporters for subcellular cell-specific targeting of anti-tumor drugs. Bioessays. 2008;30(3):278-287.

15. Gilyazova DG, Rosenkranz AA, Gulak PV, et al. Targeting cancer cells by novel engineered modular transporters. Cancer Res. 2006; 66(21):10534-10540.

16. Rosenkranz AA, Lunin VG, Gulak PV, et al. Recombinant modular transporters for cell-specific nuclear delivery of locally acting drugs enhance photosensitizer activity. FASEB J. 2003;17(9): 1121-1123.

17. Slastnikova TA, Rosenkranz AA, Gulak PV, et al. Modular nanotransporters: a multipurpose in vivo working platform for targeted drug delivery. Int J Nanomed. 2012;7:467-482.

18. Koumarianou E, Slastnikova TA, Pruszynski M, et al. Radiolabeling and in vitro evaluation of ${ }^{67} \mathrm{Ga}$-NOTA-modular nanotransporter - a potential Auger electron emitting EGFR-targeted radiotherapeutic. Nucl Med Biol. 2014;41(6):441-449.

19. Rosenkranz AA, Vaidyanathan G, Pozzi OR, Lunin VG, Zalutsky MR, Sobolev AS. Engineered modular recombinant transporters: application of new platform for targeted radiotherapeutic agents to alphaparticle emitting ${ }^{211}$ At. Int J Radiat Oncol Biol Phys. 2008;72(1): 193-200.

20. Slastnikova TA, Koumarianou E, Rosenkranz AA, et al. Modular nanotransporters: a versatile approach for enhancing nuclear delivery and cytotoxicity of Auger electron-emitting ${ }^{125}$ I. EJNMMI Res. 2012;2(1):59.

21. Slastnikova TA, Rosenkranz AA, Morozova NB, et al. Preparation, cytotoxicity and in vivo antitumor efficacy of ${ }^{111}$ In-labeled modular nanotransporters. Int J Nanomedicine. 2017;12:395-410.

22. Leamon CP, Low PS. Delivery of macromolecules into living cells: a method that exploits folate receptor endocytosis. Proc Natl Acad Sci US A. 1991;88(13):5572-5576.

23. Sun Y, Martell AE, Welch MJ. Synthesis of a Newdiaminodithiolbifunctionalchelator for radiolabeling biomolecules with indium (III). Tetrahedron. 2000;56(29):5093-5103.

24. Hather G, Liu R, Bandi S, et al. Growth rate analysis and efficient experimental design for tumor xenograft studies. Cancer Inform. 2014; 13 (Suppl 4):65-72. 
25. Edelman MJ, Harb WA, Pal SE, et al. Multicenter trial of EC145 in advanced, folate-receptor positive adenocarcinoma of the lung. J Thorac Oncol. 2012;7(10):1618-1621.

26. Leamon CP, Reddy JA, Vlahov IR, et al. Preclinical antitumor activity of a novel folate-targeted dual drug conjugate. Mol Pharm. 2007; 4(5):659-667.

27. Siegel BA, Dehdashti F, Mutch DG, et al. Evaluation of 111 In-DTPAfolate as a receptor-targeted diagnostic agent for ovarian cancer: initial clinical results. J Nucl Med. 2003;44(5):700-707.

28. Leamon CP, Parker MA, Vlahov IR, et al. Synthesis and biological evaluation of EC20: a new folate-derived, $(99 \mathrm{~m}) \mathrm{Tc}-$ based radiopharmaceutical. Bioconjug Chem. 2002;13(6):1200-1210.

29. Hoogstins CE, Tummers QR, Gaarenstroom KN, et al. A novel tumorspecific agent for intraoperative near-infrared fluorescence imaging: a translational study in healthy volunteers and patients with ovarian cancer. Clin Cancer Res. 2016;22(12):2929-2938.

30. Lee RJ, Low PS. Folate-mediated tumor cell targeting of liposomeentrapped doxorubicin in vitro. Biochim Biophys Acta. 1995; 1233(2):134-144.

31. Zhang Y, Zhou J, Yang C, et al. Folic acid-targeted disulfide-based cross-linking micelle for enhanced drug encapsulation stability and site-specific drug delivery against tumors. Int J Nanomedicine. 2016; 11:1119-1130.

32. Porta F, Lamers GE, Morrhayim J, et al. Folic acid-modified mesoporous silica nanoparticles for cellular and nuclear targeted drug delivery. Adv Healthc Mater. 2013;2(2):281-286.

33. Guo X, Shi C, Wang J, Di S, Zhou S. pH-triggered intracellular release from actively targeting polymer micelles. Biomaterials. 2013;34(18):4544-4554.

34. Chen C, Ke J, Zhou XE, et al. Structural basis for molecular recognition of folic acid by folate receptors. Nature. 2013;500(7463):486-489.

35. Lee RJ, Low PS. Delivery of liposomes into cultured KB cells via folate receptor-mediated endocytosis. J Biol Chem. 1994;269(5): 3198-3204.
36. Sonvico F, Dubernet C, Marsaud V, et al. Establishment of an in vitro model expressing the folate receptor for the investigation of targeted delivery systems. J Drug Delivery Sci Technol. 2005;15(6):407-410.

37. Xu L, Kittrell S, Yeudall WA, Yang H. Folic acid-decorated polyamidoaminedendrimer mediates selective uptake and high expression of genes in head and neck cancer cells. Nanomedicine (Lond). 2016;11(22):2959-2973.

38. Li G, Wang Y, Huang K, Zhang H, Peng W, Zhang C. The experimental study on the radioimmunotherapy of the nasopharyngeal carcinoma overexpressing HER2/neu in nude mice model with intratumoral injection of ${ }^{188}$ Re-herceptin. Nucl Med Biol. 2005;32(1):59-65.

39. Liu W, MacKay JA, Dreher MR, et al. Injectable intratumoral depot of thermally responsive polypeptide-radionuclide conjugates delays tumor progression in a mouse model. J Control Release. 2010;144(1):2-9.

40. Celikoglu F, Celikoglu SI, York AM, Goldberg EP. Intratumoral administration of cisplatin through a bronchoscope followed by irradiation for treatment of inoperable non-small cell obstructive lung cancer. Lung Cancer. 2006;51(2):225-236.

41. Nakase Y, Hagiwara A, Kin S, et al. Intratumoral administration of methotrexate bound to activated carbon particles: antitumor effectiveness against human colon carcinoma xenografts and acute toxicity in mice. J Pharmacol Exp Ther. 2004;311(1):382-387.

42. van Herpen CM, van der Laak JA, de Vries IJ, et al. Intratumoral recombinant human interleukin-12 administration in head and neck squamous cell carcinoma patients modifies locoregional lymph node architecture and induces natural killer cell infiltration in the primary tumor. Clin Cancer Res. 2005;11(5):1899-1909.

43. Nag S, Erickson B, Thomadsen B, et al. The American Brachytherapy Society recommendations for high-dose-rate brachytherapy for carcinoma of the cervix. Int J Radiat Oncol Biol Phys. 2000;48(1): 201-211. 


\section{Supplementary materials Optimization of reaction conditions for conjugation of Mal-PEG-FA to MNT}

The site-specific conjugation of maleimide (Mal) polyethylene glycol (PEG) folic acid (FA) (Mal-PEG-FA) to modular nanotransporter (MNT) turned out to be a somewhat tricky step. At first, we revealed that in contrast to the manufacturer's claims, tris(2-carboxyethyl)phosphine hydrochloride (TCEP) used for preliminary SH-reduction interfered with the maleimide reaction; however, this appeared to be quite consistent with previously published data. ${ }^{1,2}$ We got low yield (24\%) when performing model $\mathrm{NH}_{2}$-PEG-Mal conjugation to MNT in the presence of $5 \mathrm{mM}$ TCEP. The addition of a rather brief dialysis step following 40 min SH-reduction and decreasing final TCEP concentration to $8.2 \mu \mathrm{M}$ in the reaction mixture resulted in significant increase in reaction yield $(87 \%)$. In parallel studies, we compared TCEP with another reducing agent - 1,4-dithiothreitol (DTT). Here, TCEP turned out to give a better yield $(55 \% \pm 5 \%$ for TCEP vs $34 \% \pm 7 \%$ for DTT) of subsequent maleimide reaction under these specific reaction conditions (not optimized otherwise) (Figure S1A). Thus, all further optimizations were performed using TCEP for reduction of MNT SH-groups. Finally, we tested different FA-PEG-Mal:MNT molar ratios to find the optimum. As anticipated, the reaction yield increased with increase in FA-PEG-Mal:MNT molar ratio starting from 19\% (2:1) to $82 \%$ (50:1) (Figure S1B). However, further increase in FA-PEG-Mal:MNT molar ratio, though almost vanishing the band corresponding to non-modified MNT, led to formation of high molecular weight products appearing as bands at the top of the sodium dodecyl sulfate polyacrylamide gel electrophoresis (SDS-PAGE) gel (Figure S1C). These high molecular weight bands are most likely due to cross-linked conjugates of several MNT molecules, presumably to some trace impurities in FA-PEG-Mal. Based on the dependence of reaction yield (excluding high molecular products) of FA-PEG-Mal:MNT molar ratio (Figure S1D), we chose 50-fold molar excess of FA-PEG-Mal as the optimal ratio for subsequent routine MNT modification.

\section{Confocal laser scanning microscopy imaging of FR-positive cells after incubation with MNT-PEG-FA}

HeLa (FR-positive) cells were seeded on coverslips in 24 -well plates $\left(9 \times 10^{4}\right.$ cells/well). The next day, the medium was changed to fresh medium containing $1 \%$ bovine serum albumin (BSA). Then NOTA-MNT-PEG-FA was added to the wells to final concentration of $50 \mathrm{nM}$, and the cells were incubated for $48 \mathrm{~h}$ at $37^{\circ} \mathrm{C}$ in a $5 \% \mathrm{CO}_{2}$ humidified atmosphere. Afterward, medium containing unbound MNT was removed and the cells were washed four times with Hanks solution followed by acetone-methanol (1:1) fixation for $30 \mathrm{~min}$ at $-40^{\circ} \mathrm{C}$. Then the cells were air-dried, and the plate was closed tightly and stored at $-40^{\circ} \mathrm{C}$ until immunocytochemical staining and imaging. Prior to imaging, the cells were permeabilized in phosphate-buffered saline (PBS) with $0.1 \%$ Tween- 20 and $0.2 \%$ Triton X-100 for $35 \mathrm{~min}$ at $37^{\circ} \mathrm{C}$, stained with primary rabbit anti-MNT antibodies $(\mathrm{Ab})$, followed by staining with secondary goat anti-rabbit $\mathrm{Ab}$ conjugated with Alexa Fluor 555 (Invitrogen, Carlsbad,

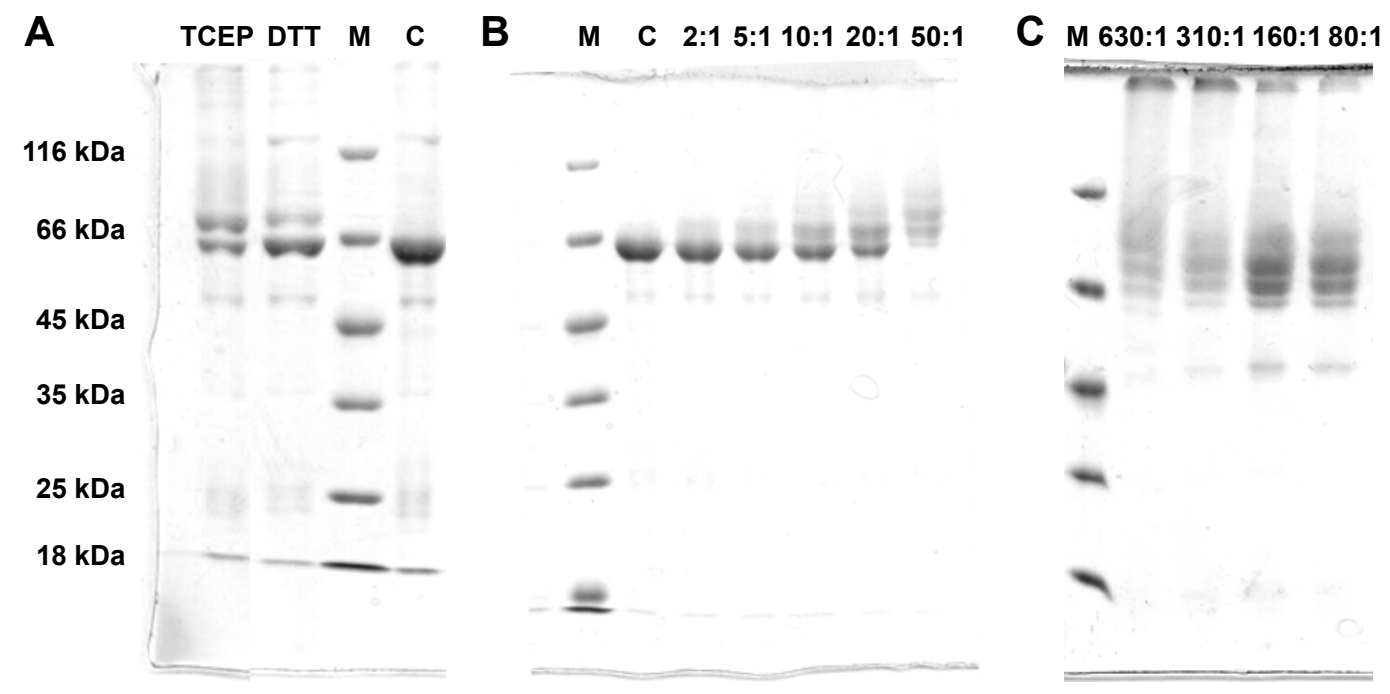

Figure SI (Continued) 


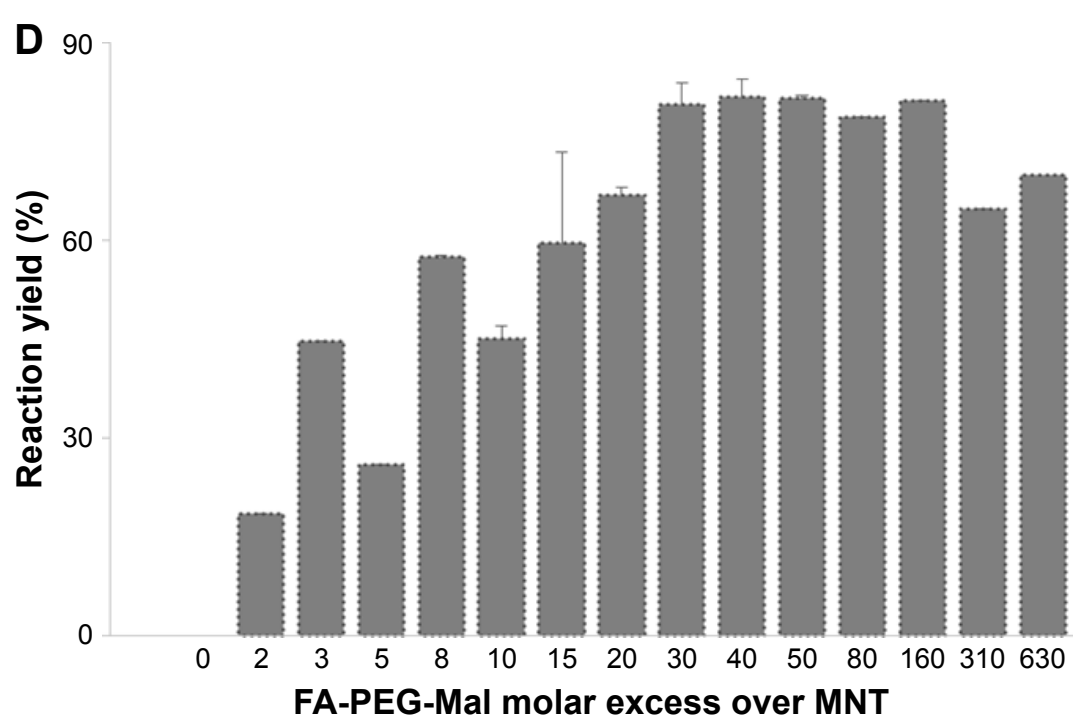

Figure SI Optimization of reaction conditions for conjugation of Mal-PEG-FA to MNT.

Notes: (A) SDS-PAGE gel under nonreducing conditions of MNT (designated as C) and MNT reduced by either TCEP or DTT followed by subsequent conjugation with $\mathrm{NH}_{2}$-PEG-FA in 10:I molar excess of the latter. (B) SDS-PAGE gel under nonreducing conditions of MNT (designated as C) and MNT reduced by TCEP, followed by subsequent conjugation with Mal-PEG-FA in 2:1; 5:I; 10:1; 20:1; 50:1 molar excess of the latter. (C) SDS-PAGE gel under nonreducing conditions of MNT reduced by TCEP, followed by subsequent conjugation with Mal-PEG-FA in 80:1; 160:1; 310:1; 630:1 molar excess of the latter. M corresponds to molecular weight protein ladder. (D) The dependence of reaction yield of Mal-PEG-FA conjugation to MNT from Mal-PEG-FA molar excess over MNT. Error bars represent standard errors of mean.

Abbreviations: DTT, I,4-dithiothreitol; FA, folic acid; Mal, maleimide; MNTs, modular nanotransporters; PEG, polyethylene glycol; SDS-PAGE, sodium dodecyl sulfate polyacrylamide gel electrophoresis; TCEP, tris(2-carboxyethyl)phosphine hydrochloride.

CA), then with tertiary rabbit anti-goat Ab conjugated with Alexa Fluor 555, followed by staining with 4',6-diamidino-2phenylindole (DAPI) (Sigma) and mounted on a glass microscope slide using Shandon Immu-mount mounting media (Thermo Scientific, Pittsburg, PA). Subcellular localization

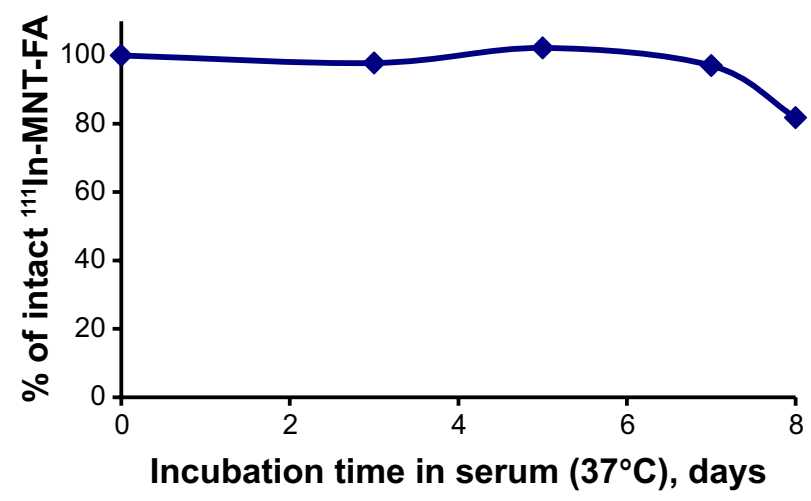

Figure S2 Stability of "'In-MNT-FA. 2-3 $\mu$ L aliquots of "'In-NOTA-MNT were mixed with $7-8 \mu \mathrm{L}$ of calf fetal serum at $37^{\circ} \mathrm{C}$ for up to 8 days. After incubation, the aliquots were analyzed by Laemmli sodium dodecyl sulfate polyacrylamide gel electrophoresis using Mini-Protean TGX Any kD gels.

Abbreviations: FA, folic acid; MNTs, modular nanotransporters. was investigated using an LSM 510 META NLO multiphoton laser scanning microscope (Carl Zeiss) equipped with a Mai Tai Broadband Mode-Locked Ti-sapphire Laser (SpectraPhysics) with Plan-Apochromat $\times 63 / 1.4$ Oil DIC lens. DAPI fluorescence was two-photon excited with a Ti-sapphire

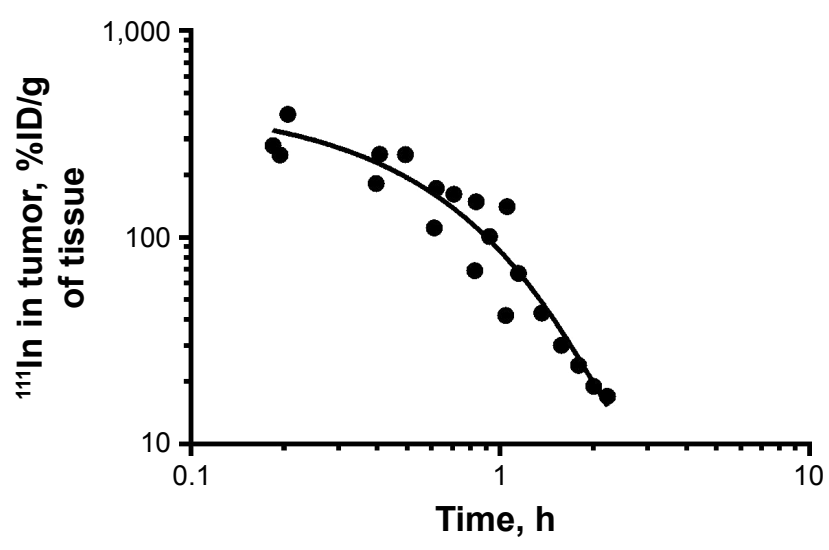

Figure S3 Serial single-photon emission computed tomography/CT imaging of nude mice bearing HeLa human cervical carcinoma xenografts after intratumoral injection of control "'IIIn.

Notes: Decay-corrected clearance of '"'In activity from tumor following control II'In injection. The solid line represents a fit of the data to a monoexponential model. 


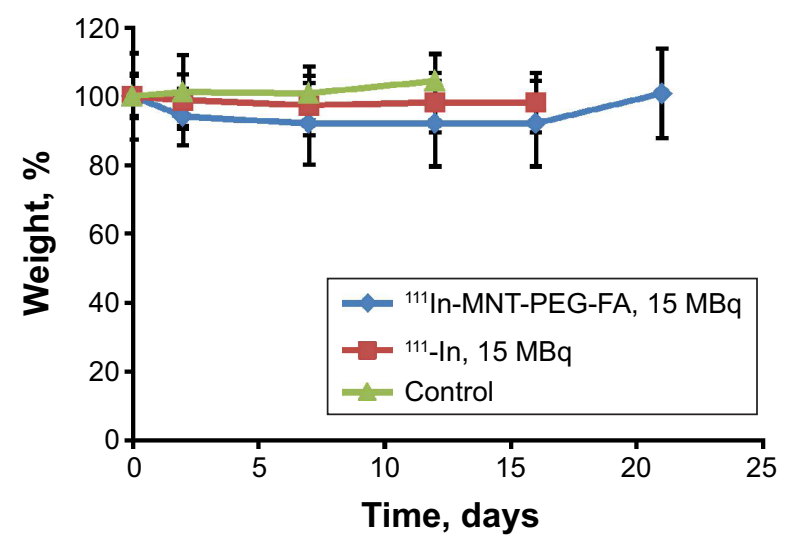

Figure S4 Dependence of the weight of HeLa-bearing mice following a single intratumoral injection of "'In-MNT-PEG-FA (I5 MBq/7.I $\mu$ g), control "''In (I5 MBq), or unlabeled MNT-PEG-FA (7.I $\mu \mathrm{g})$ designated as Control.

Abbreviations: FA, folic acid; MNTs, modular nanotransporters; PEG, polyethylene glycol.

Laser (790 nm), while Alexa Fluor 555 fluorescence was excited with a He-Ne laser (543 nm).

To assess nonspecific uptake, parallel wells with coverslips with FA-containing media $(1 \mathrm{mM})$ were processed in the same way. To determine cell autofluorescence, the addition of NOTA-MNT-PEG-FA was omitted, and the coverslips were processed in the same way.
The typical images obtained by confocal laser scanning microscopy are presented in Figure S5, demonstrating the ability of NOTA-MNT-PEG-FA to reach the nuclei of FR-positive HeLa cells. The process is FR-specific, as the presence of $1 \mathrm{mM} F A$ in the incubation media diminished the signal significantly (Figure S5).
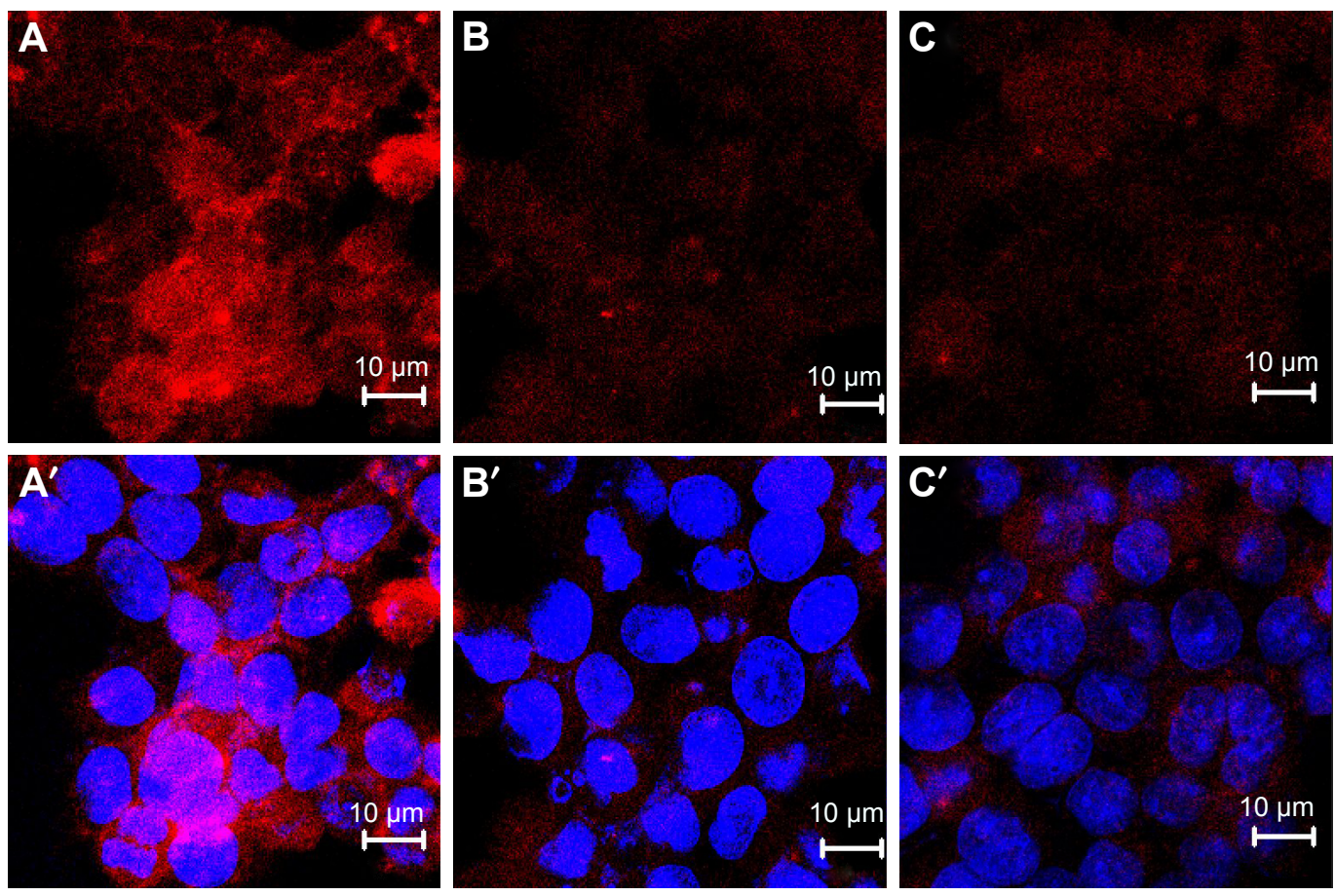

Figure S5 Intracellular distribution of NOTA-MNT-PEG-FA visualized by confocal laser scanning microscopy.

Notes: Confocal laser scanning microscopy images (slices made across the nuclei) of HeLa (FR-positive) cells incubated with either 50 nM NOTA-MNT-PEG-FA alone $\left(\mathbf{A}, \mathbf{A}^{\prime}\right)$ or in the presence I mM FA (B, B') for $48 \mathrm{~h}$. Untreated cells served as autofluorescence controls (C, C'). NOTA-MNT-PEG-FA is visualized by immunocytochemical staining using secondary and tertiary antibodies conjugated with Alexa Fluor 555. Alexa Fluor 555 fluorescence corresponding to NOTA-MNT-PEG-FA is in red pseudocolor $(\mathbf{A}-\mathbf{C})$, and overlay of Alexa Fluor 555 fluorescence and 4',6-diamidino-2-phenylindole staining for nuclei is presented in blue pseudo-color $\left(\mathbf{A}^{\prime}-\mathbf{C}^{\prime}\right)$. Bar $=10 \mu \mathrm{m}$. Plan-Apochromat $\times 63 / 1.4$ Oil DIC lens, zoom $2 \times$.

Abbreviations: FA, folic acid; MNTs, modular nanotransporters; PEG, polyethylene glycol. 


\section{References}

1. Tyagarajan K, Pretzer E, Wiktorowicz JE. Thiol-reactive dyes for fluorescence labeling of proteomic samples. Electrophoresis. 2003;24(14): $2348-2358$.

2. Shafer DE, Inman JK, Lees A. Reaction of Tris(2-carboxyethyl)phosphine (TCEP) with maleimide and $\alpha$-haloacyl groups: anomalous elution of TCEP by gel filtration. Anal Biochem. 2000;282(1):161-164.

\section{Publish your work in this journal}

Drug Design, Development and Therapy is an international, peerreviewed open-access journal that spans the spectrum of drug design and development through to clinical applications. Clinical outcomes, patient safety, and programs for the development and effective, safe, and sustained use of medicines are the features of the journal, which

Submit your manuscript here: http://www.dovepress.com/drug-design-development-and-therapy-journal has also been accepted for indexing on PubMed Central. The manuscript management system is completely online and includes a very quick and fair peer-review system, which is all easy to use. Visit http://www.dovepress.com/testimonials.php to read real quotes from published authors. 